\title{
A combinatorial method for computing characteristic polynomials of starlike hypergraphs
}

\author{
Yan-Hong Bao ${ }^{1} \cdot$ Yi-Zheng Fan $^{1}$ (1) $\cdot$ Yi Wang $^{1} \cdot$ Ming Zhu $^{2}$ \\ Received: 18 September 2018 / Accepted: 2 April 2019 / Published online: 22 May 2019 \\ (c) Springer Science+Business Media, LLC, part of Springer Nature 2019
}

\begin{abstract}
Using the Poisson formula for resultants, and variants of the chip-firing game on graphs, we provide a combinatorial method for computing a class of resultants corresponding to the characteristic polynomials of the adjacency tensors of starlike hypergraphs including hyperpaths and hyperstars, which are given recursively and explicitly.
\end{abstract}

Keywords Resultant · Hypergraph · Characteristic polynomial · Adjacency tensor · Chip firing

Mathematics Subject Classification $05 \mathrm{C} 65 \cdot 13 \mathrm{P} 15 \cdot 15 \mathrm{~A} 18$

\section{Introduction}

Here, a tensor (or hypermatrix) refers to a multi-array of entries in some field, which can be viewed to be the coordinates of the classical tensor (as multilinear function)

This work was supported by National Natural Science Foundation of China (Grant Nos. 11871071, 11871073, 11771016).

$\bowtie \quad$ Yi-Zheng Fan

fanyz@ahu.edu.cn

Yan-Hong Bao

baoyh@ahu.edu.cn

Yi Wang

wangy@ahu.edu.cn

Ming Zhu

zhuming@ahu.edu.cn

1 School of Mathematical Sciences, Anhui University, Hefei 230601, People's Republic of China

2 School of Electronics and Information Engineering, Anhui University, Hefei 230601,

People's Republic of China 
under an orthonormal basis. The eigenvalues of a tensor were introduced by Qi $[17,18]$ and Lim [14] independently. To find the eigenvalues of a tensor, Qi [17,18] introduced the characteristic polynomial of a tensor, which is defined to be a resultant of a system of homogeneous polynomials. In general, there is not an explicit polynomial formula yet for resultants except in some very special cases; and many fundamental questions about resultants still remain open.

To our knowledge, there are currently three tools to compute a concrete resultant. The first one is Koszul complex, whose terms are given by the graded tensor product of a polynomial algebra and an exterior algebra, and the differential is built from objective polynomials in the resultant. The resultant is exactly equal to a certain characteristic of the related Koszul complex, see $[1,16]$. The second one is the generalized trace, which is defined by Morozov and Shakirov [15]. Using the generalized traces and the Schur function, Cooper and Dutle [5] expressed the characteristic polynomial of a hypergraph and characterized several properties of the characteristic polynomial, including the characteristic polynomial for a single edge. Hu et al. [12] gave an expression of the characteristic polynomial of a tensor. Shao, Qi and Hu [19] gave a graph theoretic formula for the generalized trace. The third tool is the Poisson formula, which may provide an inductive computing method, see [11, Chapter 13, Theorem 1.2] or [13, Proposition 2.7]. For example, Cooper and Dutle computed the spectrum of the "all ones" tensors using the Poisson formula, see [6, Theorem 3]. We refer to [11, Chapter 13] and [7, Chapter 3] for an overview of calculation of resultants.

Recently, spectral hypergraph theory has been developed to explore connections between the structure of a uniform hypergraph and the eigenvalues of some related symmetric tensors. Cooper and Dutle [5] proposed the concept of the adjacency tensor for a uniform hypergraph. Shao et al. [20] proved that the adjacency tensor of a connected $k$-uniform hypergraph $G$ has a symmetric H-spectrum if and only if $k$ is even and $G$ is odd-Obipartite. This result gives a method to check whether a connected even-uniform hypergraph is odd-bipartite or not. Fan et al. $[9,10]$ use the spectral symmetry or the eigenvariety associated with spectral radius of the adjacency tensor to characterize the structural properties of hypergraphs.

The characteristic polynomial of a hypergraph is defined to be the characteristic polynomial of its adjacency tensor. In this paper, we mainly aim to give a lower dimension formula to compute the characteristic polynomial of hypergraphs based on the Poisson formula and variants of the chip-firing game, and determine the characteristic polynomials of starlike hypergraphs including hyperstars and hyperpaths, recursively and explicitly.

For simplicity of notation, we denote $[n]=\{1,2, \ldots, n\}$ and $[m, n]=\{m, m+$ $1, \ldots, n\}$ for integers $m<n$.

\section{Preliminaries}

In this section, we recall some basic notions and useful results on resultants and hypergraphs. 


\subsection{Resultants}

Let $F_{1}\left(x_{1}, \ldots, x_{n}\right), \ldots, F_{n}\left(x_{1}, \ldots, x_{n}\right)$ be $n$ homogeneous polynomials over $\mathbb{C}$ in variables $x_{1}, \ldots, x_{n}$, where the degree of $F_{i}$ is $d_{i}>0$ for $i \in[n]$. An important question is whether the system of equations

$$
\left\{\begin{array}{l}
F_{1}\left(x_{1}, \ldots, x_{n}\right)=0 \\
\cdots \\
F_{n}\left(x_{1}, \ldots, x_{n}\right)=0
\end{array}\right.
$$

admits nontrivial solutions.

Generally, each $F_{i}$ can be written as

$$
F_{i}=\sum_{|\alpha|=d_{i}} c_{i, \alpha} \mathbf{x}^{\alpha}
$$

where $\alpha=\left(i_{1}, \ldots, i_{n}\right),|\alpha|=i_{1}+\cdots+i_{n}$ and $\mathbf{x}^{\alpha}=x_{1}^{i_{1}} \cdots x_{n}^{i_{n}}$. Note that the number of $\alpha$ 's with $|\alpha|=d$ is $\left(\begin{array}{c}n+d-1 \\ d\end{array}\right)$.

For each possible pair of indices $i, \alpha$, we introduce a variable $u_{i, \alpha}$. Then, given a polynomial $P \in \mathbb{C}\left[u_{i, \alpha}:|\alpha|=d_{i}, i \in[n]\right]$, we let $P\left(F_{1}, \ldots, F_{n}\right)$ denote the value obtained by replacing each variable $u_{i, \alpha}$ in $P$ with the corresponding coefficient $c_{i, \alpha}$.

Theorem 2.1 [7, Chapter 3, Theorem 2.3] For fixed positive degrees $d_{1}, \ldots, d_{n}$, there exists a unique polynomial $\operatorname{Res} \in \mathbb{Z}\left[u_{i, \alpha}\right]$ satisfying the following properties:

(i) If $F_{1}, \ldots, F_{n} \in \mathbb{C}\left[x_{1}, \ldots, x_{n}\right]$ are homogeneous of degrees $d_{1}, \ldots, d_{n}$, respectively, the system (2.1) has a nontrivial solution if and only if $\operatorname{Res}\left(F_{1}, \ldots, F_{n}\right)=$ 0 .

(ii) $\operatorname{Res}\left(x_{1}^{d_{1}}, \ldots, x_{n}^{d_{n}}\right)=1$.

(iii) Res is irreducible, even regarded as a polynomial in $\mathbb{C}\left[u_{i, \alpha}\right]$.

$\operatorname{Res}\left(F_{1}, \ldots, F_{n}\right)$ is called the resultant of $F_{1}, \ldots, F_{n}$. Resultants have important applications in algebraic geometry, algebraic combinatorics and spectral hypergraph theory. However, it is difficult to compute the resultant of general polynomials. Here, we list some useful properties and calculation methods of resultants which will be used in this paper.

Lemma $2.2\left[5\right.$, Lemma 3.2] Let $F_{1}, \ldots, F_{n} \in \mathbb{C}\left[x_{1}, \ldots, x_{n}\right]$ be homogeneous polynomials of degree $d_{1}, \ldots, d_{n}$, respectively, and let $G_{1}, \cdots, G_{m} \in \mathbb{C}\left[y_{1}, \ldots, y_{m}\right]$ be homogeneous polynomials of degree $\delta_{1}, \ldots, \delta_{m}$, respectively. Then,

$$
\operatorname{Res}\left(F_{1}, \ldots, F_{n}, G_{1}, \ldots, G_{m}\right)=\operatorname{Res}\left(F_{1}, \ldots, F_{n}\right)^{\prod_{j=1}^{m} \delta_{j}} \operatorname{Res}\left(G_{1}, \ldots, G_{m}\right)^{\prod_{i=1}^{n} d_{i}}
$$


Lemma $2.3[7$, Chapter 3, Theorem 3.1] For a fixed $j \in[n]$ and $\lambda \in \mathbb{C}$,

$$
\operatorname{Res}\left(F_{1}, \ldots, F_{j-1}, \lambda F_{j}, F_{j+1}, \ldots, F_{n}\right)=\lambda^{d_{1} \cdots d_{j-1} d_{j+1} \cdots d_{n}} \operatorname{Res}\left(F_{1}, \ldots, F_{n}\right),
$$

where $d_{i}$ is the degree of $F_{i}$ for each $i \in[n]$.

Next, we recall the Poisson formula. Given homogeneous polynomials $F_{1}, \ldots, F_{n} \in$ $\mathbb{C}\left[x_{1}, \ldots, x_{n}\right]$ of degree $d_{1}, \ldots, d_{n}$, respectively, let

$$
\begin{array}{r}
f_{i}\left(x_{1}, \ldots, x_{n-1}\right)=F_{i}\left(x_{1}, \ldots, x_{n-1}, 1\right), \quad(1 \leq i \leq n), \\
\bar{F}_{i}\left(x_{1}, \ldots, x_{n-1}\right)=F_{i}\left(x_{1}, \ldots, x_{n-1}, 0\right), \quad(1 \leq i \leq n-1)
\end{array}
$$

Observe that $\bar{F}_{1}, \ldots, \bar{F}_{n-1}$ are still homogeneous in $\mathbb{C}\left[x_{1}, \ldots, x_{n-1}\right]$ of degree $d_{1}, \ldots, d_{n-1}$, respectively, but $f_{1}, \ldots, f_{n}$ are not homogeneous in general.

Lemma 2.4 [Poisson formula] Keep the above notation. If $\operatorname{Res}\left(\bar{F}_{1}, \ldots, \bar{F}_{n-1}\right) \neq 0$, then the quotient algebra $A=\frac{\mathbb{C}\left[x_{1}, \ldots, x_{n-1}\right]}{\left\langle f_{1}, \ldots, f_{n-1}\right\rangle}$ has dimension $d_{1} \cdots d_{n-1}$ as a vector space over $\mathbb{C}$, where $\left\langle f_{1}, \ldots, f_{n-1}\right\rangle$ is the ideal of the polynomial algebra $C\left[x_{1}, \ldots, x_{n-1}\right]$ generated by $f_{1}, \ldots, f_{n-1}$, and

$$
\operatorname{Res}\left(F_{1}, \ldots, F_{n}\right)=\operatorname{Res}\left(\bar{F}_{1}, \ldots, \bar{F}_{n-1}\right)^{d_{n}} \operatorname{det}\left(m_{f_{n}}: A \rightarrow A\right),
$$

where $m_{f_{n}}: A \rightarrow A$ is the multiplication map given by $f_{n}$.

Here, the above form of the Poisson formula follows from [7, Chapter 3, Theorem 3.4], which is different from the original one in [13].

\subsection{Hypergraphs}

A hypergraph $H$ is a pair $(V, E)$, where $V$ is the set of vertices and $E \subset \mathcal{P}(V)$ is the set of edges. A hypergraph $H$ is called $k$-uniform for an integer $k \geq 2$ if for each $\varepsilon \in E,|\varepsilon|=k$. Clearly, a 2-uniform hypergraph is just a classical simple graph.

Definition 2.5 [5] Let $H=(V, E)$ be a $k$-uniform hypergraph. The (normalized) adjacency tensor $\mathcal{A}(H)=\left(a_{i_{1} \cdots i_{k}}\right)_{i_{1}, \cdots, i_{k} \in V}$ is defined by

$$
a_{i_{1} \cdots i_{k}}= \begin{cases}\frac{1}{(k-1) !}, & \text { if }\left\{i_{1}, \ldots, i_{k}\right\} \in E, \\ 0, & \text { otherwise. }\end{cases}
$$

For convenience, we use the following notation. Let $V$ be a finite set and $m$ a positive integer. For each $\mathbf{e}=\left(i_{1}, \ldots, i_{m}\right) \in V^{m}$ and $\mathbf{c}=\left(c_{1}, \ldots, c_{m}\right) \in \mathbb{N}^{m}$, we denote $\mathbf{x}_{\mathbf{e}}^{\mathbf{c}}=x_{i_{1}}^{c_{1}} \cdots x_{i_{m}}^{c_{m}}$. We also write $\mathbf{x}_{\mathbf{e}}^{\mathbb{1}}$ as $\mathbf{x}_{\mathbf{e}}$, where $\mathbb{1}=(1, \ldots, 1) \in \mathbb{N}^{m}$. If $V=[n], \mathbf{c}=\left(c_{1}, \ldots, c_{n}\right) \in \mathbb{N}^{n}$, we write $\mathbf{x}_{[n]}^{\mathbf{c}}$ as $\mathbf{x}^{\mathbf{c}}$.

The eigenvalues of a tensor were introduced by Qi [17,18] and Lim [14] independently. The adjacency tensor of a uniform hypergraph was introduced by Cooper and 
Dutle [5]. Here, we briefly give the definition of eigenvalues of uniform hypergraphs based on the above.

Definition 2.6 [5,17] Let $H=(V, E)$ be a $k$-uniform hypergraph and $\mathcal{A}=\left(a_{i_{1} \cdots i_{k}}\right)$ be the adjacency tensor of $H$. For some $\lambda \in \mathbb{C}$, if there exists a nonzero vector $\mathbf{x} \in \mathbb{C}^{|V|}$ such that for each $j \in V$,

$$
\sum_{i_{2}, i_{3}, \ldots, i_{k} \in V} a_{j i_{2} i_{3} \ldots i_{k}} x_{i_{2}} x_{i_{3}} \cdots x_{i_{k}}=\lambda x_{j}^{k-1},
$$

or equivalently, for each $v \in V$,

$$
\sum_{v \in \varepsilon \in E} \mathbf{x}_{\varepsilon \backslash\{v\}}=\lambda x_{v}^{k-1},
$$

then $\lambda$ is called an eigenvalue of $H$.

For each $v \in V$, define

$$
F_{v}=\lambda x_{v}^{k-1}-\sum_{v \in \varepsilon \in E} \mathbf{x}_{\varepsilon \backslash\{v\}} .
$$

The polynomial

$$
\phi_{H}(\lambda)=\operatorname{Res}\left(F_{v}: v \in V\right)
$$

in the indeterminant $\lambda$ is called the characteristic polynomial of $H$. Consequently, $\lambda$ is an eigenvalue of $H$ if and only if $\phi_{H}(\lambda)=0$.

\subsection{The dollar game on graphs}

Let $G=(V, E)$ be a simple graph. Recall that a configuration $\mathbf{c}$ on $G$ means a function c $: V \rightarrow \mathbb{N}$, which can be understood as a pile of $\mathbf{c}(v)$ tokens (chips, or dollars) at each vertex $v$. A dollar game on $G$ starts from a configuration c. At each step of the game, a vertex $v$ is fired, that is, dollars move from $v$ to its adjacent vertices, one dollar going along each edge incident to $v$. Fix a vertex $w$ of $G$, called the bank vertex. A vertex $v$ other than $w$ can be fired if and only if $\mathbf{c}(v) \geq \operatorname{deg}(v)$, where $\operatorname{deg}(v)$ is the degree of the vertex $v$. The bank vertex $w$ is allowed to go into debt such that $w$ can be fired if and only if no other firing is possible.

Suppose that $\mathcal{X}$ is a non-empty finite sequence of (not necessarily distinct) vertices of $G$, such that starting from a configuration $\mathbf{c}$, the vertices can be fired in the order of $\mathcal{X}$. If $v$ occurs $x(v)$ times, we shall refer to $x$ as the representative vector for $\mathcal{X}$. The configuration $\mathbf{c}^{\prime}$ after the sequence of firing $\mathcal{X}$ is given by

$$
\mathbf{c}^{\prime}=\mathbf{c}-L x,
$$

where $L$ is the Laplacian matrix of $G$. 
The dollar game on graphs was introduced by Biggs [2] and is a variant of the chip-firing game. It is often described in terms of "snowfalls" and "avalanches" in the literature. A configuration $\mathbf{c}$ is said to be stable if $0 \leq \mathbf{c}(v)<\operatorname{deg}(v)$ for every $v \neq w$. A sequence of firing is $w$-legal if and only if each occurrence of a vertex $v \neq w$ follows a configuration $t$ with $t(v) \geq \operatorname{deg}(v)$ and each occurrence of $w$ follows a stable configuration. A configuration $\mathbf{c}$ on $G$ is said recurrent if there is a $w$-legal sequence for $\mathbf{c}$ which leads to the same configuration. A critical configuration $\mathbf{c}$ means that $\mathbf{c}$ is both stable and recurrent. We refer to [2] for more details.

Lemma 2.7 [2, Theorem 6.2] If $G$ is a connected graph, then the number of critical configurations is equal to the number of spanning trees of $G$.

Example 2.8 Let $K_{k}$ be a complete graph on $k$ vertices. Then, the number of critical configurations is $k^{k-2}$.

\section{Poisson formula for characteristic polynomials of hypergraphs}

\subsection{Poisson formula for hypergraphs}

Let $H=(V, E)$ be a $k$-uniform hypergraph. Recall that the characteristic polynomial of $H$ is defined as

$$
\phi_{H}(\lambda)=\operatorname{Res}\left(F_{v}: v \in V\right)
$$

where

$$
F_{v}=\lambda x_{v}^{k-1}-\sum_{v \in \varepsilon \in E} \mathbf{x}_{\varepsilon \backslash\{v\}} \in \mathbb{C}\left[x_{v}: v \in V\right] .
$$

In order to use Poisson formula for the resultant $\operatorname{Res}\left(F_{v}: v \in V\right)$, we need fix a vertex $w$ in $V$. Denote by $E_{w}$ the set of all edges containing the vertex $w$ and $\varepsilon_{\widehat{w}}=\varepsilon \backslash\{w\}$ for each $\varepsilon \in E_{w}$. Then, we have

$$
f_{w}=\lambda-\sum_{\varepsilon \in E_{w}} \mathbf{x}_{\varepsilon_{\widehat{w}}},
$$

and for all $v \in V \backslash\{w\}$,

$$
\begin{aligned}
& f_{v}=\lambda x_{v}^{k-1}-\sum_{v \in \varepsilon \in E \backslash E_{w}} \mathbf{x}_{\varepsilon \backslash\{v\}}-\sum_{v \in \varepsilon \in E_{w}} \mathbf{x}_{\varepsilon_{w} \backslash\{v\}}, \\
& \bar{F}_{v}=\lambda x_{v}^{k-1}-\sum_{v \in \varepsilon \in E \backslash E_{w}} \mathbf{x}_{\varepsilon \backslash\{v\}},
\end{aligned}
$$

where $f_{w}$ and $f_{v}$ are obtained from $F_{w}$ and $F_{v}$ by taking $x_{w}=1$, respectively, and $\bar{F}_{v}$ is obtained from $F_{v}$ by taking $x_{w}=0$. 
Deleting the vertex $w$ in $V$ and the edges in $E_{w}$, one can obtain a sub-hypergraph $\widehat{H}=(\widehat{V}, \widehat{E})$. To be precise, $\widehat{V}=V \backslash\{w\}$ and $\widehat{E}=E \backslash E_{w}$.

Lemma 3.1 Retain the above notation. Then,

$$
\phi_{H}(\lambda)=\phi_{\widehat{H}}(\lambda)^{k-1} \operatorname{det}\left(m_{f_{w}}: A \rightarrow A\right),
$$

where $A$ is the quotient algebra $\frac{\mathbb{C}\left[x_{v}: v \in \widehat{V}\right]}{\left\langle f_{v}: v \in \widehat{V}\right\rangle}$ and $m_{f_{w}}$ is the multiplication map of A given by $f_{w}$.

Proof By Lemma 2.4, the characteristic polynomial of $H$ is

$$
\phi_{H}(\lambda)=\operatorname{Res}\left(\bar{F}_{v}: v \in \widehat{V}\right)^{k-1} \operatorname{det}\left(m_{f_{w}}: A \rightarrow A\right) .
$$

Considering the sub-hypergraph $\widehat{H}$ of $H$, by Eq. (3.1), we have

$$
\phi_{\widehat{H}}(\lambda)=\operatorname{Res}\left(\bar{F}_{v}: v \in \widehat{V}\right) .
$$

The result follows.

By definition, the algebra $A$ is $(k-1)^{r-1}$-dimensional as a vector space over $\mathbb{C}$ where $r$ is the number of vertices of $H$. In general, it is difficult to compute the determinant $\operatorname{det}\left(m_{f_{w}}: A \rightarrow A\right)$. However, we can give a description for some special cases.

\subsection{Hypergraphs with a cut vertex}

Let $H=(V, E)$ be a $k$-uniform connected hypergraph and $w \in V$. Denote $E_{\widehat{w}}=\left\{\varepsilon_{\widehat{w}} \mid\right.$ $\left.\varepsilon \in E_{w}\right\}$. Deleting the vertex $w$, we can get a (non-uniform) hypergraph $\widetilde{H}=(\widetilde{V}, \widetilde{E})$, with $\widetilde{V}=\widehat{V}=V \backslash\{w\}$ and $\widetilde{E}=\left(E \backslash E_{w}\right) \cup E_{\widehat{w}}$. Recall the vertex $w$ is called a cut vertex if $\widetilde{H}$ is not connected; see Fig. 1. Suppose that $w$ is a cut vertex and $\widetilde{H}_{1}=\left(\widetilde{V}_{1}, \widetilde{E}_{1}\right), \cdots, \widetilde{H}_{n}=\left(\widetilde{V}_{n}, \widetilde{E}_{n}\right)(n \geq 2)$ are the connected components of $\widetilde{H}$. For each $i \in[n]$, we set $V_{i}=\widetilde{V}_{i}, E_{i}=\widetilde{E}_{i} \backslash E_{\widehat{w}}$, and then obtain a sub-hypergraph $H_{i}=\left(V_{i}, E_{i}\right)$ of $H$. Note that each $H_{i}$ is a $k$-uniform hypergraph and may not be connected.

For each $i \in[n]$, we denote

$$
E_{w}^{i}=\left\{\varepsilon \in E_{w} \mid \varepsilon \cap V_{i} \neq \varnothing\right\}
$$

By definition, we have

$$
\phi_{H}(\lambda)=\operatorname{Res}\left(F_{v}: v \in V\right),
$$


Fig. 1 A $k$-uniform hypergraph $H$ with a cut vertex $w$

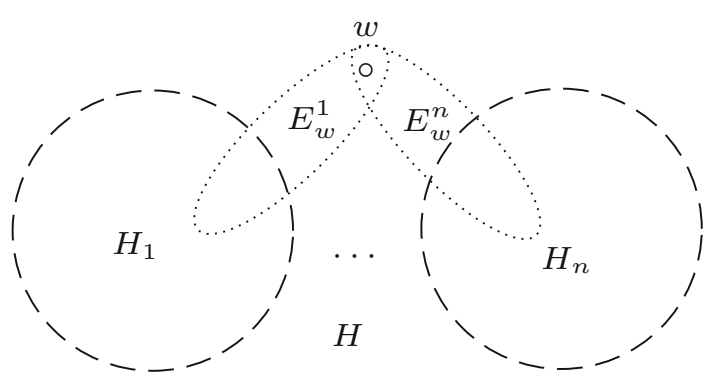

where

$$
F_{w}=\lambda x_{w}^{k-1}-\sum_{i=1}^{n} \sum_{\varepsilon \in E_{w}^{i}} \mathbf{x}_{\varepsilon_{\widehat{w}}}
$$

and for all $v_{i} \in V_{i}, i \in[n]$,

$$
F_{v_{i}}=\lambda x_{v_{i}}^{k-1}-\sum_{v_{i} \in \varepsilon \in E_{i}} \mathbf{x}_{\varepsilon \backslash\left\{v_{i}\right\}}-\sum_{v_{i} \in \varepsilon \in E_{w}^{i}} \mathbf{x}_{\varepsilon \backslash\left\{v_{i}\right\}},
$$

Therefore,

$$
f_{w}=\lambda-\sum_{i=1}^{n} \sum_{\varepsilon \in E_{w}^{i}} \mathbf{x}_{\varepsilon_{\widehat{w}}}
$$

and for all $v_{i} \in V_{i}, i \in[n]$,

$$
\begin{aligned}
& f_{v_{i}}=\lambda x_{v_{i}}^{k-1}-\sum_{v_{i} \in \varepsilon \in E_{i}} \mathbf{x}_{\varepsilon \backslash\left\{v_{i}\right\}}-\sum_{v_{i} \in \varepsilon \in E_{w}^{i}} \mathbf{x}_{\varepsilon_{\widehat{w}} \backslash\left\{v_{i}\right\}}, \\
& \bar{F}_{v_{i}}=\lambda x_{v_{i}}^{k-1}-\sum_{v_{i} \in \varepsilon \in E_{i}} \mathbf{x}_{\varepsilon \backslash\left\{v_{i}\right\}} .
\end{aligned}
$$

Let $A$ be the quotient algebra $\frac{\mathbb{C}\left[x_{v}: v \in \widehat{V}\right]}{\left\langle f_{v}: v \in \widehat{V}\right\rangle}$, and let $m_{f_{w}}: A \rightarrow A$ be the multiplication map given by $f_{w}$. Since $V_{1}, \ldots, V_{n}$ form a partition of $\widehat{V}$ and for each $v_{i} \in V_{i}, f_{v_{i}} \in \mathbb{C}\left[x_{v}: v \in V_{i}\right]$, each monomial of $A$ can be expressed as $\mathbf{x}_{\widehat{V}}^{\mathbf{c}}=\mathbf{x}_{V_{1}}^{\mathbf{c}_{1}} \mathbf{x}_{V_{2}}^{\mathbf{c}_{2}} \cdots \mathbf{x}_{V_{n}}$ by considering $f_{v}=0$ inside $A$ for $v \in V_{i}$ and $i \in[n]$. So we have $A=A_{1} \otimes \cdots \otimes A_{n}$, where

$$
A_{i}=\frac{\mathbb{C}\left[x_{v}: v \in V_{i}\right]}{\left\langle f_{v}: v \in V_{i}\right\rangle}, i \in[n] .
$$

We define and denote $m_{i, w}: A_{i} \rightarrow A_{i}$ the linear map given by the multiplication by $\sum_{\varepsilon \in E_{w}^{i}} \mathbf{x}_{\varepsilon_{\widehat{w}}}$ for each $i \in[n]$. Then, 


$$
m_{f_{w}}=\lambda \operatorname{Id}_{A}-\sum_{i=1}^{n} \operatorname{Id}_{A_{1}} \otimes \cdots \otimes \operatorname{Id}_{A_{i-1}} \otimes m_{i, w} \otimes \operatorname{Id}_{A_{i+1}} \otimes \cdots \otimes \operatorname{Id}_{A_{n}},
$$

where Id denotes the identity map on certain vector space.

Assumption 1 For each $i \in[n]$, there exists an ordered $\mathbb{C}$-basis $\mathbf{x}^{\alpha_{i, 1}}, \ldots, \mathbf{x}^{\alpha_{i, d_{i}}}$ for $A_{i}$ such that the matrix of $m_{i, w}$ with respect to this basis is a lower triangular matrix with the diagonal entry $\alpha_{i, j_{i}}, j_{i} \in\left[d_{i}\right]$, where $d_{i}=(k-1)^{r_{i}}$ and $r_{i}=\left|V_{i}\right|$.

Under Assumption $1,\left\{\mathbf{x}^{\alpha_{1, j_{1}}} \cdots \mathbf{x}^{\alpha_{n, j_{n}}} \mid j_{i} \in\left[d_{i}\right], i \in[n]\right\}$ with the left lexicographic order is a basis for $A$ such that the matrix of $m_{f_{w}}$ with respect to this basis is still a lower triangular matrix with diagonal entries $\lambda-\sum_{1 \leq i \leq n} \alpha_{i, j_{i}}$ for $j_{i} \in\left[d_{i}\right]$ and $i \in[n]$. In this situation, we have

$$
\operatorname{det}\left(m_{f_{w}}: A \rightarrow A\right)=\prod_{\substack{1 \leq j_{i} \leq d_{i} \\ 1 \leq i \leq n}}\left(\lambda-\sum_{i=1}^{n} \alpha_{i, j_{i}}\right)
$$

Corollary 3.2 Let $H$ be a k-uniform hypergraph with a cut vertex $w$. Then, under Assumption 1,

$$
\phi_{H}(\lambda)=\prod_{i=1}^{n} \phi_{H_{i}}(\lambda)^{(k-1)^{1+\sum_{j \neq i} r_{j}}} \prod_{\substack{1 \leq j_{i} \leq d_{i} \\ 1 \leq i \leq n}}\left(\lambda-\sum_{i=1}^{n} \alpha_{i, j_{i}}\right) .
$$

Proof By definition, the characteristic polynomial of $H_{i}=\left(V_{i}, E_{i}\right)$ is

$$
\phi_{H_{i}}(\lambda)=\operatorname{Res}\left(\bar{F}_{v_{i}}: v_{i} \in V_{i}\right)
$$

By Lemma 2.2, we have

$$
\operatorname{Res}\left(\bar{F}_{v}: v \in \widehat{V}\right)=\prod_{i=1}^{n} \operatorname{Res}\left(\bar{F}_{v_{i}}: v_{i} \in V_{i}\right)^{(k-1)^{\sum_{j \neq i}\left|V_{j}\right|}}=\prod_{i=1}^{n} \phi_{H_{i}}(\lambda)^{(k-1)^{\sum_{j \neq i} r_{j}}} .
$$

From Lemma 3.1, it follows that

$$
\begin{aligned}
& \phi_{H}(\lambda)=\operatorname{Res}\left(\bar{F}_{v}: v \in \hat{V}\right)^{k-1} \operatorname{det}\left(m_{f_{w}}: A \rightarrow A\right) \\
& =\prod_{i=1}^{n} \phi_{H_{i}}(\lambda)^{(k-1)^{1+\sum_{j \neq i} r_{j}}} \prod_{\substack{j_{i} \in\left[d_{i}\right] \\
i \in[n]}}\left(\lambda-\sum_{i=1}^{n} \alpha_{i, j_{i}}\right) \text {. }
\end{aligned}
$$


Fig. 2 A hypergraph $H$ with a cored vertex $w$

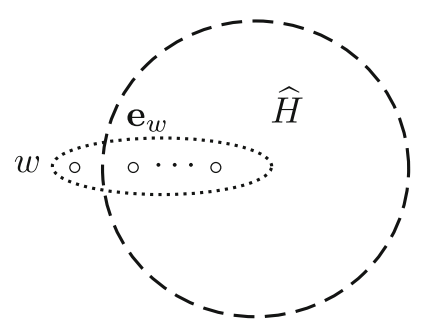

\subsection{Hypergraphs with a cored vertex}

Let $H=(V, E)$ be a $k$-uniform hypergraph. Recall that a vertex $w \in V$ is called a cored vertex if it is contained in only one edge; see Fig. 2. Deleting the cored vertex $w$ and the edge $\varepsilon_{w}$ containing $w$, one can obtain a sub-hypergraph $\widehat{H}=(\widehat{V}, \widehat{E})$ with $\widehat{V}=V \backslash\{w\}$ and $\widehat{E}=E \backslash\left\{\varepsilon_{w}\right\}$. Then,

$$
\begin{aligned}
& F_{w}=\lambda x_{w}^{k-1}-\mathbf{x}_{\varepsilon_{\widehat{w}}}, \\
& F_{v}=\lambda x_{v}^{k-1}-\sum_{v \in \varepsilon \in \widehat{E}} \mathbf{x}_{\varepsilon \backslash\{v\}}-\sum_{v \in \varepsilon_{w}} \mathbf{x}_{\varepsilon_{w} \backslash\{v\}}, v \neq w .
\end{aligned}
$$

Moreover, $f_{w}=\lambda-\mathbf{x}_{\varepsilon_{\widehat{w}}}$, and for all $v \neq w$,

$$
\begin{aligned}
& f_{v}=\lambda x_{v}^{k-1}-\sum_{v \in \varepsilon \in \widehat{E}} \mathbf{x}_{\varepsilon \backslash\{v\}}-\sum_{v \in \varepsilon_{w}} \mathbf{x}_{\varepsilon_{\widehat{w}} \backslash\{v\}}, \\
& \bar{F}_{v}=\lambda x_{v}^{k-1}-\sum_{v \in \varepsilon \in \widehat{E}} \mathbf{x}_{\varepsilon \backslash\{v\}} .
\end{aligned}
$$

Corollary 3.3 Let $H$ be a $k$-hypergraph with a cored vertex $w$. Retain the above notation. Then,

$$
\phi_{H}(\lambda)=\phi_{\widehat{H}}(\lambda)^{k-1} \operatorname{det}\left(m_{f_{w}}: A \rightarrow A\right),
$$

where $A$ is the quotient algebra $\frac{\mathbb{C}\left[x_{v}: v \in \widehat{V}\right]}{\left\langle f_{v}: v \in \widehat{V}\right\rangle}$ and $m_{f_{w}}$ is the multiplication map of A given by $f_{w}$.

\section{Hyperpaths}

Let $P_{n}^{k}$ be a $k$-uniform hyperpath with $n$ edges or of length $n$, which has vertices labeled as $0,1, \ldots, r=n(k-1)$ from left to right as in Fig. 3, and edges $\varepsilon_{t}=$ $\{(k-1)(t-1),(k-1)(t-1)+1, \ldots,(k-1) t\}$ for $t \in[n]$.

In this section, we will give a recursive formula for the characteristic polynomials of hyperpaths. By the Poisson formula introduced in Lemma 3.1, it suffices to compute 

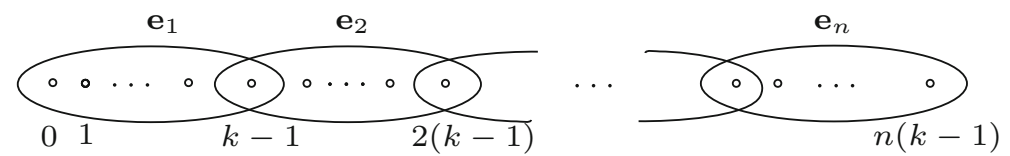

Fig. 3 A $k$-uniform hyperpath $P_{n}^{k}$ with $n$ edges

the related determinant. For this, we introduce the following model of the dollar game on hypergraphs.

\subsection{The dollar game on hypergraphs and the firing graph}

We now define a dollar game on a hypergraph, considered as a variant of the dollar game on a graph. Let $H=(V, E)$ be a $k$-uniform hypergraph with a specified bank vertex $w$. A function $\mathbf{c}: V \rightarrow \mathbb{N}$ is called a configuration on $H$. A dollar game starts from a configuration $\mathbf{c}$. At each of step of the game, a vertex $v$ is fired on an edge $\varepsilon$ containing $v$, that is, the vertex $v$ decreases $k-1$ dollars, and each of the vertices of $\varepsilon$ other than $v$ increases 1 dollar, which yields a new configuration on $H$. Note that such $v$ will be fired on all edges containing $v$, one edge each step, which yields $\operatorname{deg}(v)$ new configurations. A vertex $v$ other than $w$ can be fired if and only if $\mathbf{c}(v) \geq k-1$. The bank vertex $w$ can be fired regardless of the value $\mathbf{c}(w)$, but only when no other vertex can be fired. We say that a configuration $\mathbf{c}$ is stable if $0 \leq \mathbf{c}(v)<k-1$ for all $v \neq w$. The above setting of the dollar game on hypergraphs is different from that of the dollar game on simple graphs in Sect. 2.3, but will be useful for our discussion.

Let " $\leq$ " be a total ordering on the set $\widehat{V}=V \backslash\{w\}$. Let $\mathbf{c}$ be a configuration on $H$. The weight of $\mathbf{c}$ is defined and denoted to be $\omega(\mathbf{c})=\sum_{v \in \widehat{V}} \mathbf{c}(v)$. We define the left anti-lexicographical order $\prec$ for all configurations on $H$. To be precise, for any configurations $\mathbf{c}$ and $\mathbf{c}^{\prime}, \mathbf{c} \prec \mathbf{c}^{\prime}$ if and only if either $\omega(\mathbf{c})<\omega\left(\mathbf{c}^{\prime}\right)$, or $\omega(\mathbf{c})=\omega\left(\mathbf{c}^{\prime}\right)$, $\mathbf{c}(i)=\mathbf{c}^{\prime}(i)$ for any $1 \leq i \leq t-1$ and $\mathbf{c}(t)>\mathbf{c}^{\prime}(t)$ for some $t$.

Based on the above discussion, we now define a directed graph, called a firing graph $\mathcal{G}\left(\mathbf{c}_{0}\right)$ associated with a stable configuration $\mathbf{c}_{0}$ on the hypergraph $H$, which is closely related to the dollar game on $H$ starting from $\mathbf{c}_{0}$. Here, a vertex of $\mathcal{G}\left(\mathbf{c}_{0}\right)$ is a configuration and a directed edge will be called an arrow.

Step 1 Initially, set $\mathcal{V}_{0}=\left\{\mathbf{c}_{0}\right\}$ and $\mathcal{E}_{0}=\varnothing$.

Step 2 For each edge $\varepsilon$ containing $w$, let $\overline{\mathbf{c}}_{0, \varepsilon}$ be a configuration given by

$$
\overline{\mathbf{c}}_{0, \varepsilon}(v)= \begin{cases}\mathbf{c}_{0}(v)+1, & \text { if } v \in \varepsilon \backslash\{w\}, \\ \mathbf{c}_{0}(v), & \text { otherwise. }\end{cases}
$$

Put $\mathcal{V}_{1}=\mathcal{V}_{0} \cup\left\{\overline{\mathbf{c}}_{0, \varepsilon}: w \in \varepsilon\right\}$ and $\mathcal{E}_{1}=\left\{\mathbf{c}_{0} \stackrel{\alpha_{w, \varepsilon}}{\longrightarrow} \overline{\mathbf{c}}_{0, \varepsilon}: w \in \varepsilon\right\}$, where the arrow $\alpha_{w, \varepsilon}$ means that the configuration $\overline{\mathbf{c}}_{0, \varepsilon}$ is obtained from $\mathbf{c}_{0}$ by firing the bank vertex $w$ on the edge $\varepsilon$.

Step 3 If all of configurations in $\mathcal{V}_{i} \backslash \mathcal{V}_{i-1}$ are stable, then we define $\mathcal{V}\left(\mathcal{G}\left(\mathbf{c}_{0}\right)\right)=\mathcal{V}_{i}$ and $\mathcal{E}\left(\mathcal{G}\left(\mathbf{c}_{0}\right)\right)=\mathcal{E}_{i}$. Otherwise, for each non-stable configuration $\mathbf{c} \in \mathcal{V}_{i} \backslash \mathcal{V}_{i-1}$, we 


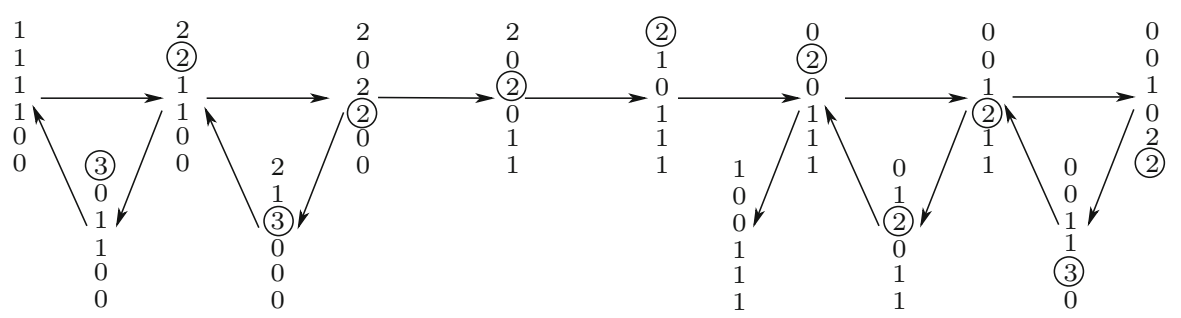

Fig. 4 Firing graph of $P_{3}^{3}$ associated with $\mathbf{c}_{0}$

choose the vertex

$$
u_{\mathbf{c}}:=\max \{v \in \widehat{V} \mid \mathbf{c}(v) \geq k-1\}
$$

For each edge $\varepsilon \in E$ containing $u_{\mathbf{c}}$, we define an arrow $\mathbf{c} \stackrel{\alpha_{u_{\mathbf{c}}, \varepsilon}}{\longrightarrow} \overline{\mathbf{c}}_{\varepsilon}$, where the configuration $\overline{\mathbf{c}}_{\varepsilon}$ is given by

$$
\overline{\mathbf{c}}_{\varepsilon}(v)= \begin{cases}\mathbf{c}(v)-(k-1), & \text { if } v=u_{\mathbf{c}}, \\ \mathbf{c}(v)+1, & \text { if } v \in \varepsilon \backslash\left\{u_{\mathbf{c}}\right\} \\ \mathbf{c}(v), & \text { otherwise }\end{cases}
$$

and

$$
\begin{aligned}
& \mathcal{V}_{i+1}=\mathcal{V}_{i} \cup\left\{\overline{\mathbf{c}}_{\varepsilon} \mid u_{\mathbf{c}} \in \varepsilon \in E, \mathbf{c} \text { is not stable in } \mathcal{V}_{i} \backslash \mathcal{V}_{i-1}\right\}, \\
& \mathcal{E}_{i+1}=\mathcal{E}_{i} \cup\left\{\mathbf{c} \stackrel{\alpha_{u_{\mathbf{c}}, \varepsilon}}{\longrightarrow} \overline{\mathbf{c}}_{\varepsilon} \mid u_{\mathbf{c}} \in \varepsilon \in E, \mathbf{c} \text { is not stable in } \mathcal{V}_{i} \backslash \mathcal{V}_{i-1}\right\}
\end{aligned}
$$

Note that $\overline{\mathbf{c}}_{\varepsilon}$ may have been in $\mathcal{V}_{i}$. If $\overline{\mathbf{c}}_{\varepsilon} \notin \mathcal{V}_{i}$, we say that $\overline{\mathbf{c}}_{\varepsilon}$ is obtained from $\mathbf{c}$ by firing $u_{\mathbf{c}}$ on $\varepsilon$.

Step 3 tells us the firing rule, that is, which vertex will be fired at the next step among all non-stable vertices other than the bank vertex. We use the maximal principle, the non-stable vertex with the maximal order will be firstly fired. In addition, from the construction of $\mathcal{G}\left(\mathbf{c}_{0}\right)$, we have $0 \leq \omega(\mathbf{c}) \leq \omega\left(\mathbf{c}_{0}\right)+k-1$ for any $\mathbf{c} \in \mathcal{V}\left(\mathcal{G}\left(\mathbf{c}_{0}\right)\right)$, which implies that $\mathcal{G}\left(\mathbf{c}_{0}\right)$ is a finite directed graph.

Example 4.1 Let $P_{3}^{3}$ be the 3-uniform hyperpath with 3 edges as in Fig. 3 by taking $n=3$ and $k=3$. Let 0 be the bank vertex of $P_{3}^{3}$, and let $\mathbf{c}_{0}=(*, 1,1,1,1,0,0)$. Here, the value of the bank vertex 0 is omitted in each configuration of the dollar game starting from $\mathbf{c}_{0}$. The firing graph $\mathcal{G}\left(\mathbf{c}_{0}\right)$ is drawn in Fig. 4, where each vertex within a circle means that it will be fired at the next step.

Generally speaking, it is difficult to obtain the firing graphs $\mathcal{G}\left(\mathbf{c}_{0}\right)$ for all stable configurations $\mathbf{c}_{0}$ on a general hypergraph. However, for some special classes of hypergraphs, e.g., hyperpaths, we can characterize the structure of $\mathcal{G}\left(\mathbf{c}_{0}\right)$. 


\subsection{Firing graphs of hyperpaths}

In this part, we characterize the structure of the firing graph of $P_{n}^{k}$ in Fig. 3 associated with the arbitrary fixed stable configuration, where the vertex 0 is the bank vertex. For a configuration $\mathbf{c}$ on $[0, n(k-1)]$, we denote $\mathbf{c}=\left(\mathbf{c}(0), \mathbf{c}^{1}, \ldots, \mathbf{c}^{n}\right)$, where $\mathbf{c}^{i}$ is the restriction of $\mathbf{c}$ on $\widehat{\varepsilon}_{i}:=\varepsilon_{i} \backslash\{(i-1)(k-1)\}$, i.e.,

$$
\mathbf{c}^{i}=(\mathbf{c}((i-1)(k-1)+1), \ldots, \mathbf{c}(i(k-1))), i \in[n] .
$$

Let $\tilde{\mathbf{c}}^{i}$ be the restriction of $\mathbf{c}$ on $\varepsilon_{i}$, i.e.,

$$
\tilde{\mathbf{c}}^{i}=(\mathbf{c}((i-1)(k-1)), \mathbf{c}((i-1)(k-1)+1), \ldots, \mathbf{c}(i(k-1))), i \in[n] .
$$

Then, for each $i \in[n], \tilde{\mathbf{c}}^{i}$ can be considered as a stable configuration on the complete graph $K_{k}$ with the vertex set $\varepsilon_{i}$ and the bank vertex $(i-1)(k-1)$.

Let $\mathcal{S}_{k}$ be the set of all stable configurations on the complete graph $K_{k}$, and $\mathcal{C}_{k}$ be the set of all critical configurations on $K_{k}$. Denote by $\mathcal{B}$ the set of all stable configurations on $P_{n}^{k}$, and for $s \in[0, n-1]$

$\mathcal{B}_{s}=\left\{\left(\mathbf{c}^{1}, \ldots, \mathbf{c}^{n}\right) \mid \tilde{\mathbf{c}}^{i} \in \mathcal{C}_{k}\right.$ for $i \in[s], \tilde{\mathbf{c}}^{s+1} \notin \mathcal{C}_{k}$, and $\tilde{\mathbf{c}}^{i} \in \mathcal{S}_{k}$ for $\left.i \in[s+1, n]\right\}$

and $\mathcal{B}_{n}=\left\{\left(\mathbf{c}^{1}, \ldots, \mathbf{c}^{n}\right) \mid \tilde{\mathbf{c}}^{i} \in \mathcal{C}_{k}, i \in[n]\right\}$. Note that the bank vertex in the graph dollar game for every edge other than the first edge inherits its stability with respect to hypergraph dollar game from the stability in the graph dollar game for the previous edge. So, $\mathcal{B}$ is the disjoint union of $\mathcal{B}_{0}, \ldots, \mathcal{B}_{n}$.

Lemma 4.2 Suppose that $\mathbf{c}_{0}$ is a stable configuration on $P_{n}^{k}$, the labeled $k$-uniform hyperpath described in the opening of Sect. 4 , with 0 as the bank vertex, and $\mathcal{G}\left(\mathbf{c}_{0}\right)$ is the firing graph of $P_{n}^{k}$ associated with $\mathbf{c}_{0}$. Then,

(i) for any $t \in[n]$, if $\mathbf{c}(t(k-1))=k-1$, then $\mathbf{c}(v)<k-1$ for any $v>t(k-1)$.

(ii) for any $t \in[n]$, the vertex $t(k-1)$ is fired at $\mathbf{c} \in \mathcal{V}\left(\mathcal{G}\left(\mathbf{c}_{0}\right)\right)$ if and only if $\mathbf{c}(t(k-1))=k-1$.

(iii) for any configuration $\mathbf{c} \in \mathcal{V}\left(\mathcal{G}\left(\mathbf{c}_{0}\right)\right), \omega(\mathbf{c}) \geq \omega\left(\mathbf{c}_{0}\right)$, where the equality holds if and only if the vertices $1, \ldots, k-1$ have been fired on $\varepsilon_{1}$ exactly once along any directed path from $\mathbf{c}_{0}$ to $\mathbf{c}$.

Proof (i) Assume to the contrary, there exists a configuration $\mathbf{c}$ such that $\mathbf{c}(t(k-1))=$ $k-1$, and $\mathbf{c}(v) \geq k-1$ for some $v>t(k-1)$. According to the construction of firing graphs, there exists a directed path $P\left(\mathbf{c}_{0}, \mathbf{c}\right)$ from $\mathbf{c}_{0}$ to $\mathbf{c}$ (without repeated vertices on the path). We also assume that $\mathbf{c}$ is the first occurrence of the configuration with the above property, so that along the path $P\left(\mathbf{c}_{0}, \mathbf{c}\right)$, except $\mathbf{c}$ no configurations of $P\left(\mathbf{c}_{0}, \mathbf{c}\right)$ hold the above property.

Case 1: $t(k-1)$ is not fired before $\mathbf{c}$ along the path $P\left(\mathbf{c}_{0}, \mathbf{c}\right)$. Then, $\mathbf{c}(v)=\mathbf{c}_{0}(v)<$ $k-1$ for any $v>t(k-1)$ since such $v$ cannot gain more dollars before $t(k-1)$ is fired, a contradiction. 
Case 2: $t(k-1)$ is fired at some configuration say $\mathbf{c}_{-1}^{\prime}$ before $\mathbf{c}$ along the path $P\left(\mathbf{c}_{0}, \mathbf{c}\right)$. We may assume $t(k-1)$ is last fired at $\mathbf{c}_{-1}^{\prime}$ before $\mathbf{c}$. Consider the directed path $P\left(\mathbf{c}_{0}, \mathbf{c}\right)$ :

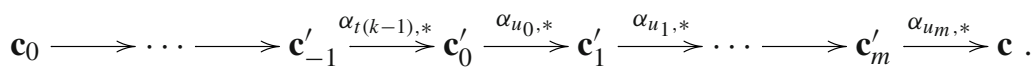

Note that $\mathbf{c}_{-1}^{\prime}(t(k-1)) \geq k-1$ and $\mathbf{c}_{-1}^{\prime}(v)<k-1$ for any $v>t(k-1)$ by the firing rule. If $\mathbf{c}(t(k-1)) \geq k$, there exists a configuration $\mathbf{c}^{\prime}$ before $\mathbf{c}_{-1}^{\prime}$ such that $\mathbf{c}^{\prime}(t(k-1))=k-1$ and $\mathbf{c}^{\prime}(v) \geq k-1$ for some $v>t(k-1)$, a contradiction to the assumption about $\mathbf{c}$. So $\mathbf{c}_{-1}^{\prime}(t(k-1))=k-1$, and $\mathbf{c}_{0}^{\prime}(t(k-1))=0$.

From $\mathbf{c}_{0}^{\prime}$ to $\mathbf{c}$, the vertex $t(k-1)$ obtains $k-1$ dollars by firing some vertices on $\varepsilon_{t}$ or $\varepsilon_{t+1}$. If $t(k-1)$ obtains one dollar by firing some vertex on $\varepsilon_{t}$ at configuration say $\mathbf{c}_{s}^{\prime}$, then $\mathbf{c}_{s}^{\prime}(v)<k-1$ for any $v>t(k-1)$ by the firing rule. Furthermore, such $v$ cannot gain more dollars from $\mathbf{c}_{s}^{\prime}$ to $\mathbf{c}$ as $t(k-1)$ would not be fired before $\mathbf{c}$. So, $\mathbf{c}(v)=\mathbf{c}_{s}^{\prime}(v)<k-1$ for any $v>t(k-1)$, a contradiction.

Otherwise, $t(k-1)$ obtains $k-1$ dollars only by firing vertices on $\varepsilon_{t+1}$. Then,

$$
\mathbf{c}_{0}^{\prime}(v)= \begin{cases}0, & \text { if } v=t(k-1) \\ \mathbf{c}_{-1}^{\prime}(v)+1, & \text { if } v \in[t(k-1)+1,(t+1)(k-1)], \\ \mathbf{c}_{-1}^{\prime}(v), & \text { if } v \geq(t+1)(k-1)+1\end{cases}
$$

It follows that the number of configurations in $\left\{\mathbf{c}_{i}^{\prime} \mid \alpha_{u_{i}, *}=\alpha_{u_{i}, \varepsilon_{t+1}}, i=0,1, \ldots, m\right\}$ is $k-1$ as $\mathbf{c}(t(k-1))=k-1$. Observe that for any $v \in[t(k-1)+1,(t+1)(k-1)-1]$,

$$
\mathbf{c}_{i}^{\prime}(v) \leq \mathbf{c}_{0}^{\prime}(v)+(k-2)=c_{-1}^{\prime}(v)+(k-1)<2 k-2 .
$$

It forces that each vertex in $[t(k-1)+1,(t+1)(k-1)-1]$ is fired exactly once on the edge $\varepsilon_{t+1}$ from $\mathbf{c}_{0}^{\prime}$ and $\mathbf{c}$, implying that the vertex $(t+1)(k-1)$ is also fired exactly once on $\varepsilon_{t+1}$.

Let $u_{j}$ be the vertex in $[t(k-1)+1,(t+1)(k-1)]$ last fired on $\varepsilon_{t+1}$. Then, $\mathbf{c}_{j}^{\prime}(v)<$ $k-1$ for any $v>(t+1)(k-1)$ by the firing rule. Also, we have $\mathbf{c}_{j+1}^{\prime}(t(k-1))=k-1$, $\mathbf{c}_{j+1}^{\prime}(v)<k-1$ for any $v \in[t(k-1)+1,(t+1)(k-1)]$ by the assumption on $u_{j}$, and $\mathbf{c}_{j+1}^{\prime}(v)<k-1$ for any $v>(t+1)(k-1)$ by the firing rule, which implies that $\mathbf{c}_{j+1}^{\prime}(v)<k-1$ for any $v>t(k-1)$. As $t(k-1)$ is last fired at $\mathbf{c}_{-1}^{\prime}$, we have $\mathbf{c}_{j+1}^{\prime}=\mathbf{c}$, also a contradiction.

(ii) The sufficiency of (ii) follows by the result (i) and the firing rule. For the necessity of (i), if $t(k-1)$ is fired, then $\mathbf{c}(t(k-1)) \geq k-1$. If $\mathbf{c}(t(k-1)) \geq k$, there exists a configuration $\mathbf{c}^{\prime}$ before $\mathbf{c}$ such that $\mathbf{c}^{\prime}(t(k-1))=k-1$ and $\mathbf{c}^{\prime}(v) \geq k-1$ for some $v>t(k-1)$, a contradiction to (i).

(iii) By definition, we have $\omega\left(\overline{\mathbf{c}}_{0}\right)=\omega\left(\mathbf{c}_{0}\right)+k-1$. Observe that for any arrow $\mathbf{c} \stackrel{\alpha_{u, \varepsilon_{t}}}{\longrightarrow} \mathbf{c}^{\prime}$, where $u \neq 0, \omega\left(\mathbf{c}^{\prime}\right)=\omega(\mathbf{c})-1$ if $\varepsilon_{t}=\varepsilon_{1}$, and $\omega\left(\mathbf{c}^{\prime}\right)=\omega(\mathbf{c})$ if $\varepsilon_{t} \neq \varepsilon_{1}$. Therefore, we need only show that no vertex is fired on $\varepsilon_{1}$ more than one time in any directed path starting from $\mathbf{c}_{0}$. Let 


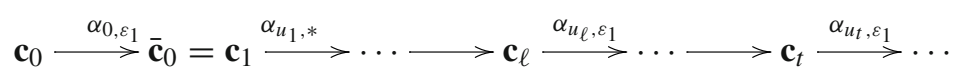

be such a walk in $\mathcal{G}\left(\mathbf{c}_{0}\right)$. If there exists a vertex in $[k-1]$ be fired more than one time on $\varepsilon_{1}$, we may assume that $u_{t}$ is the first occurrence of the vertex satisfying $\alpha_{u_{t}, \varepsilon_{1}}=\alpha_{u_{\ell}, \varepsilon_{1}}$ for some $\ell<t$. If $1 \leq u_{\ell}=u_{t} \leq k-2$, by our assumption, we have

$$
\mathbf{c}_{t}\left(u_{t}\right) \leq \overline{\mathbf{c}}_{0}\left(u_{t}\right)-(k-1)+(k-2)=\mathbf{c}_{0}\left(u_{t}\right)<k-1,
$$

since there are at most $k-2$ vertices different from $u_{t}$ in $\varepsilon_{1} \backslash\{0\}$, each fired at most once, a contradiction.

If $u_{\ell}=u_{t}=k-1$, we have $\mathbf{c}_{\ell}(v)<k-1$ for any $v>k-1$ by the firing rule, $u_{i} \in[k-2]$ for each $i \in[\ell+1, t-1]$ and each of them is fired only one time by our assumption on $u_{t}$. By the part (i), we have $\mathbf{c}_{\ell}(k-1)=k-1$. Therefore,

$$
\mathbf{c}_{t}(k-1) \leq \mathbf{c}_{\ell}(k-1)-(k-1)+(k-2)=k-2 .
$$

This is also a contradiction.

Lemma 4.3 Let $P_{1}^{k}$ be the $k$-uniform hyperpath on vertices $0,1, \ldots, k-1$ with a single edge $\varepsilon$. Let $\mathbf{c}_{0}$ be a stable configuration on $P_{1}^{k}$ with 0 as the bank vertex. Then, $\mathbf{c}_{0}$ is a critical configuration if and only if the firing graph $\mathcal{G}\left(\mathbf{c}_{0}\right)$ is a directed cycle of length $k$, and $\mathbf{c}_{0}$ is a non-critical configuration if and only if $\mathcal{G}\left(\mathbf{c}_{0}\right)$ is a directed path of length less than $k$.

Proof By the firing rule, as $P_{1}^{k}$ contains only one edge $\varepsilon$, each firing is on the edge $\varepsilon$ so that every vertex (configuration) of $\mathcal{G}\left(\mathbf{c}_{0}\right)$ has out-degree 1 . So we have a sequence of finite configurations: $\mathbf{c}_{0} \overline{\mathbf{c}}_{0} \mathbf{c}_{1} \cdots \mathbf{c}_{m}$, starting from $\mathbf{c}_{0}$ and ending at $\mathbf{c}_{m}$. Note $\omega\left(\overline{\mathbf{c}}_{0}\right)=$ $\omega\left(\mathbf{c}_{0}\right)+k-1$, and for any $\mathbf{c} \stackrel{\alpha_{u, \varepsilon_{t}}}{\longrightarrow} \mathbf{c}^{\prime}$, where $u \neq 0, \omega\left(\mathbf{c}^{\prime}\right)=\omega(\mathbf{c})-1$. So, starting from $\overline{\mathbf{c}}_{0}$, the weight of configurations in the sequence is strictly decreasing.

If $\mathbf{c}_{m}=\mathbf{c}_{0}$, or equivalently $\mathbf{c}_{0}$ is critical, then $\mathcal{G}\left(\mathbf{c}_{0}\right)$ is a directly cycle. As $\omega\left(\mathbf{c}_{m}\right)=$ $\omega\left(\mathbf{c}_{0}\right)$, by Lemma 4.2(iii), each vertex of $\varepsilon$ is fired exactly once, implying that $\mathcal{G}\left(\mathbf{c}_{0}\right)$ is a directed cycle of length $k$. Otherwise, $\mathbf{c}_{0}$ is non-critical, and $\mathcal{G}\left(\mathbf{c}_{0}\right)$ is a directed path. We assert that not all of vertices in $\varepsilon$ is fired; otherwise, by the proof of Lemma 4.2(iii), no vertex in $\varepsilon$ is fired more than once so that each of them is fired exactly once. In this case, we would get $\mathbf{c}_{m}=\mathbf{c}_{0}$, a contradiction. So $\mathcal{G}\left(\mathbf{c}_{0}\right)$ is a directed path of length less than $k$.

Remark 4.4 In Lemma 4.3, the dollar game on the hypergraph $P_{1}^{k}$ (a single edge $\varepsilon$ ) is equivalent to considering the standard dollar game on a complete (simple) graph $K_{k}$ on vertices $0,1, \ldots, k-1$ with 0 as the bank vertex. For the dollar game on simple graphs, the order of the firing can be arbitrary. However, here for dollar game on $K_{k}$, the firing order obeys the maximal principle; see Sect. 4.1.

Next, we describe the structure of the firing graph of $P_{n}^{k}$ associated with any stable configuration. 


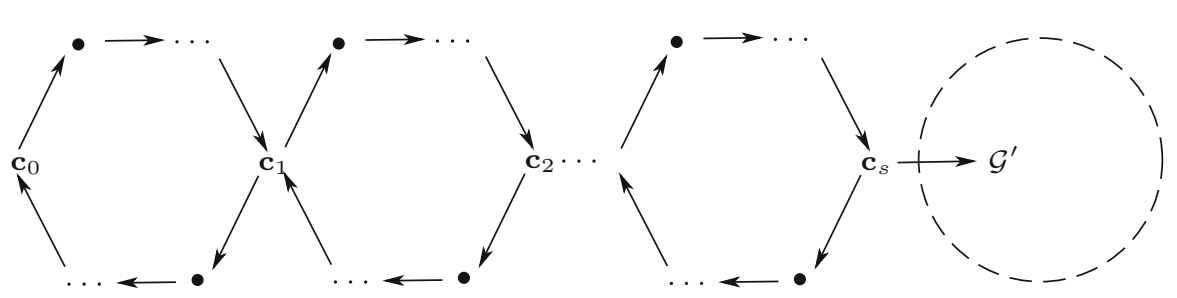

Fig. 5 Firing graph of $P_{n}^{k}$ associated with $\mathbf{c}_{0} \in \mathcal{B}_{S}$

Fig. 6 Directed cycle obtained by firing the vertices in $\varepsilon_{1}$

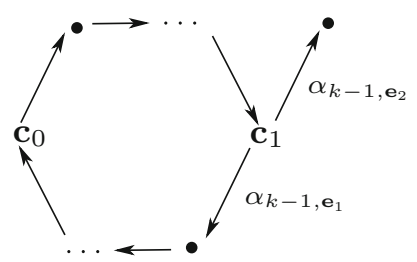

Proposition 4.5 Let $P_{n}^{k}$ be the k-uniform hyperpath as in Fig. 3. Then, for a given stable configuration $\mathbf{c}_{0} \in \mathcal{B}_{s}$ on $P_{n}^{k}$ with 0 as the bank vertex, where $s \in[n]$, the firing graph $\mathcal{G}\left(\mathbf{c}_{0}\right)$ of $P_{n}^{k}$ associated with $\mathbf{c}_{0}$ has the structure as in Fig. 5, where $\mathcal{G}^{\prime}$ is the subgraph induced by the configurations obtained by first firing the vertex $s(k-1)$ on the edge $\varepsilon_{s+1}$, each directed cycle in $\mathcal{G}\left(\mathbf{c}_{0}\right) \backslash \mathcal{G}^{\prime}$ has length $k$, such that

(i) $\mathcal{G}^{\prime}$ is not empty if and only if $s<n$;

(ii) if $s<n$, there are no arrows from $\mathcal{G}^{\prime}$ to $\mathcal{G}\left(\mathbf{c}_{0}\right) \backslash \mathcal{G}^{\prime}$;

(iii) if $s<n, \mathbf{c}_{0} \prec \mathbf{c}^{\prime}$ for any $\mathbf{c}^{\prime} \in \mathcal{G}^{\prime}$.

Proof (i) Let $\mathbf{c}_{0} \in \mathcal{B}_{s}$ be a fixed stable configuration on $P_{n}^{k}$, where $s \in[n]$. Recall that $\tilde{\mathbf{c}}_{0}^{i}$ is a stable configuration on $K_{k}$ for each $i \in[n]$, where $\tilde{\mathbf{c}}_{0}^{i}$ is defined at the beginning of this section. By definition, $\mathbf{c}_{0} \in \mathcal{B}_{s}$ means that $\tilde{\mathbf{c}}_{0}^{i} \in \mathcal{C}_{k}$ for $1 \leq i \leq s$ and $\tilde{\mathbf{c}}_{0}^{s+1} \in \mathcal{S}_{k} \backslash \mathcal{C}_{k}$. By Remark 4.4, to fire the vertices of $\varepsilon_{i}$ on the edge $\varepsilon_{i}$, it is equivalent to consider the dollar game on $K_{k}$ on vertices of $\varepsilon_{i}$ with the bank vertex $(i-1)(k-1)$, as only when $(i-1)(k-1)$ is firstly fired, the other vertices of $\varepsilon_{i}$ can gain more dollars. By Lemma 4.3, as $\tilde{\mathbf{c}}_{0}^{1}$ is a critical configuration, we get a directed cycle of length $k$ by firing the vertices $0,1, \ldots, k-1$ on $\varepsilon_{1}$ and $k-1$ also $\varepsilon_{2}$, where $\mathbf{c}_{1}$ is the configuration such that $k-1$ is a non-stable vertex with the maximal order in $\mathbf{c}_{1}$; see Fig. 6.

Similarly, since $\tilde{\mathbf{c}}_{0}^{2}, \ldots, \tilde{\mathbf{c}}_{0}^{s}$ are critical stable configurations, each $\varepsilon_{i}$ yields a directed cycle of length $k$ by firing the vertices of $\varepsilon_{i}$ on $\varepsilon_{i}, i \in[s]$. Hence, we get $s$ directed cycles, which implies that $\mathcal{G}^{\prime}$ is not empty if and only if $s<n$.

(ii) Denote

$$
\mathcal{V}^{\prime}=\left\{\mathbf{c}^{\prime} \in \mathcal{G}\left(\mathbf{c}_{0}\right) \mid \mathbf{c}^{\prime} \text { is obtained from } \mathbf{c}_{s} \text { by first firing } s(k-1) \text { on } \varepsilon_{s+1}\right\},
$$

and $\omega^{\prime}(\mathbf{c})=\sum_{v=s(k-1)+1}^{n(k-1)} \mathbf{c}(v)$ for any $\mathbf{c} \in \mathcal{G}\left(\mathbf{c}_{0}\right)$. We claim that $\omega^{\prime}\left(\mathbf{c}^{\prime}\right)>\omega^{\prime}(\mathbf{c})$ for any $\mathbf{c}^{\prime} \in \mathcal{V}^{\prime}$ and $\mathbf{c} \in \mathcal{V}\left(\mathcal{G}\left(\mathbf{c}_{0}\right)\right) \backslash \mathcal{V}^{\prime}$ 
In fact, we choose a configuration $\mathbf{c}_{m}^{\prime} \in \mathcal{V}^{\prime}$ such that $\omega^{\prime}\left(\mathbf{c}_{m}^{\prime}\right)=\min \left\{\omega^{\prime}\left(\mathbf{c}^{\prime}\right) \mid \mathbf{c}^{\prime} \in\right.$ $\left.\mathcal{V}^{\prime}\right\}$, and a directed path $P\left(\mathbf{c}_{s}, \mathbf{c}_{m}^{\prime}\right)$ from $\mathbf{c}_{s}$ to $\mathbf{c}_{m}^{\prime}$ as follows:

$$
P\left(\mathbf{c}_{s}, \mathbf{c}_{m}^{\prime}\right): \mathbf{c}_{s} \stackrel{\alpha_{s(k-1), \varepsilon_{s+1}}}{\longrightarrow} \mathbf{c}_{1}^{\prime} \stackrel{\alpha_{u_{1}^{\prime}, *}}{\longrightarrow} \cdots \longrightarrow \mathbf{c}_{m-1}^{\prime} \stackrel{\alpha_{u_{m-1}^{\prime}, *}}{\longrightarrow} \mathbf{c}_{m}^{\prime}
$$

We may also assume that $\omega^{\prime}\left(\mathbf{c}_{i}^{\prime}\right)>\omega^{\prime}\left(\mathbf{c}_{m}^{\prime}\right)$ for any $i \in[m-1]$. By the firing rule, we have $\alpha_{u_{m-1}^{\prime}, *} \neq \alpha_{u_{m-1}^{\prime}, \varepsilon_{j}}$ for any $j \in[s]$; otherwise $\omega^{\prime}\left(\mathbf{c}_{m-1}^{\prime}\right)=\omega^{\prime}\left(\mathbf{c}_{m}^{\prime}\right)$. So there exists some configurations among $\mathbf{c}_{1}^{\prime}, \ldots, \mathbf{c}_{m-1}^{\prime}$ fired on some edge $\varepsilon_{t}$ for $t \geq s+1$.

If there exists $\alpha_{u_{i}^{\prime}, *}=\alpha_{s(k-1), \varepsilon_{s+1}}$ for some $i \in[m-1]$, letting $u_{i}^{\prime}$ be the last one for the vertex $s(k-1)$ fired on $\varepsilon_{s+1}$, then it suffices to consider the restriction of the configurations on $[s(k-1), n(k-1)]$, which is equivalent to considering the firing graph of $P_{n-s}^{k}$ associated with the configuration $\left(\mathbf{c}_{i}^{\prime}(s(k-1)), \ldots, \mathbf{c}_{i}^{\prime}(n(k-1))\right)$ with $s(k-1)$ as the bank vertex, where $P_{n-s}^{k}$ is the sub-hypergraph of $P_{n}^{k}$ consisting of the edges $\varepsilon_{s+1}, \cdots, \varepsilon_{n}$. By Lemma 4.2 (iii), we have $\omega^{\prime}\left(\mathbf{c}_{m}^{\prime}\right) \geq \omega^{\prime}\left(\mathbf{c}_{i}^{\prime}\right)$, a contradiction. So, from $\mathbf{c}_{1}^{\prime}$ to $\mathbf{c}_{m}^{\prime}, s(k-1)$ is no longer fired on $\varepsilon_{s+1}$.

By the above discussion, in order to compute the $\omega^{\prime}\left(\mathbf{c}_{m}^{\prime}\right)$, it suffices to consider the firing graph of $P_{n-s}^{k}$ associated with the configuration $\left(\mathbf{c}_{s}(s(k-1)), \ldots, \mathbf{c}_{s}(n(k-1))\right)$ with $s(k-1)$ as the bank vertex. Note that $\mathbf{c}_{s}(v)=\mathbf{c}_{0}(v)=\hat{\mathbf{c}}(v)$ for any $v>s(k-1)$ and any $\hat{\mathbf{c}}$ in the front $s$ directed cycles of $\mathcal{G}\left(\mathbf{c}_{0}\right)$. By Lemma 4.2 (iii) again, we have

$$
\omega^{\prime}\left(\mathbf{c}_{m}^{\prime}\right)>\omega^{\prime}\left(\mathbf{c}_{s}\right)=\omega^{\prime}\left(\mathbf{c}_{0}\right)=\omega^{\prime}(\hat{\mathbf{c}}),
$$

since $\tilde{\mathbf{c}}_{0}^{s+1}=\left(\mathbf{c}_{0}(s(k-1)), \ldots, \mathbf{c}_{0}((s+1)(k-1))\right)$ is a non-critical. So the claim follows, and the subgraph $\mathcal{G}^{\prime}$ induced by $\mathcal{V}^{\prime}$ contains no configurations in the front $s$ directed cycles of $\mathcal{G}\left(\mathbf{c}_{0}\right)$.

(iii) We will prove the assertion by induction on $s$. If $s=0$, then Lemma 4.2(iii), $\omega\left(\mathbf{c}^{\prime}\right)>\omega\left(\mathbf{c}_{0}\right)$ for any $\mathbf{c}^{\prime}$ in $\mathcal{G}^{\prime}$ as $\tilde{\mathbf{c}}_{0}^{1}$ is not critical, implying $\mathbf{c}_{0} \prec \mathbf{c}^{\prime}$. If $s \geq 1$, also by Lemma 4.2 (iii), we have $\omega\left(\mathbf{c}^{\prime}\right) \geq \omega\left(\mathbf{c}_{0}\right)$, and $\omega\left(\mathbf{c}^{\prime}\right)=\omega\left(\mathbf{c}_{0}\right)$ if and only if the vertices $1, \ldots, k-1$ have been fired on $\varepsilon_{1}$ exactly once along any directed path from $\mathbf{c}_{0}$ to $\mathbf{c}^{\prime}$. So it suffices to consider the case of $\omega\left(\mathbf{c}^{\prime}\right)=\omega\left(\mathbf{c}_{0}\right)$.

Suppose that $P\left(\mathbf{c}_{0}, \mathbf{c}^{\prime}\right)$ is a directed path from $\mathbf{c}_{0}$ to $\mathbf{c}^{\prime}$ as follows:

$$
P\left(\mathbf{c}_{0}, \mathbf{c}^{\prime}\right): \mathbf{c}_{0} \stackrel{\alpha_{0, \varepsilon_{1}}}{\longrightarrow} \overline{\mathbf{c}}_{0}=\mathbf{c}_{1} \stackrel{\alpha_{u_{1}, *}}{\longrightarrow} \cdots \longrightarrow \mathbf{c}_{s} \stackrel{\alpha_{s(k-1), \varepsilon_{s+1}}}{\longrightarrow} \cdots \longrightarrow \mathbf{c}_{t} \stackrel{\alpha_{u_{t}, *}}{\longrightarrow} \mathbf{c}^{\prime} .
$$

As $\omega\left(\mathbf{c}^{\prime}\right)=\omega\left(\mathbf{c}_{0}\right)$, we have a unique $\sigma(v) \in[t]$ such that $\alpha_{u_{\sigma(v)}, *}=\alpha_{v, \varepsilon_{1}}$ for each $v \in[k-1]$ by Lemma 4.2 (iii). It follows that

$$
\mathbf{c}^{\prime}(v)=\mathbf{c}_{0}(v) \text { for } v \in[k-2] .
$$

By the construction of $\mathcal{G}\left(\mathbf{c}_{0}\right)$, there exist $1 \leq i_{1}<j_{1} \leq t$ such that $\alpha_{u_{i_{1}}, *}=\alpha_{k-1, \varepsilon_{2}}$ and $\alpha_{u_{j_{1}}, *}=\alpha_{k-1, \varepsilon_{1}}$. We also have

$$
\mathbf{c}_{0}(v)=\mathbf{c}_{i_{1}}(v), \mathbf{c}_{j_{1}}(v)=\mathbf{c}^{\prime}(v) \text {, for any } v>k-1 \text {. }
$$


If there exists an $\ell_{1}$ such that $i_{1}<\ell_{1}<j_{1}$ and $1 \leq u_{\ell_{1}} \leq k-2$, then by Lemma 4.2 (ii),

$$
\begin{aligned}
& \mathbf{c}_{i_{1}}(k-1)=\mathbf{c}_{0}(k-1)+1+m_{1}=k-1, \\
& \mathbf{c}_{j_{1}}(k-1)=\mathbf{c}_{\ell_{1}}(k-1)+m_{2}=k-1,
\end{aligned}
$$

where $m_{1}$ (respectively, $m_{2}$ ) is the number of vertices of [ $k-2$ ] fired on $\varepsilon_{1}$ from $\mathbf{c}_{1}$ to $\mathbf{c}_{i_{1}-1}$ (respectively, from $\mathbf{c}_{\ell_{1}}$ to $\mathbf{c}_{j_{1}-1}$ ). By the firing rule, $\mathbf{c}_{\ell_{1}}(k-1)<k-1$. So, after $\mathbf{c}_{j_{1}}$, only the vertices of $[k-2], k-1-\left(m_{1}+m_{2}+1\right)$ vertices in total, can be fired (certainly on $\varepsilon_{1}$ ). We have

$$
\begin{aligned}
\mathbf{c}^{\prime}(k-1) & =k-1-\left(m_{1}+m_{2}+1\right) \\
& =\mathbf{c}_{0}(k-1)-\left((k-1)-\mathbf{c}_{u_{\ell_{1}}}(k-1)\right)<\mathbf{c}_{0}(k-1),
\end{aligned}
$$

which implies that $\mathbf{c}_{0} \prec \mathbf{c}^{\prime}$.

Otherwise, we consider the dollar game on $P_{n-1}^{k}$, where $P_{n-1}^{k}$ is the sub-hypergraph of $P_{n}^{k}$ consisting of the edge $\varepsilon_{2}, \ldots, \varepsilon_{n}$ with $k-1$ as the bank vertex. The game starts from $\hat{\mathbf{c}}_{i_{1}}$ and produces a sequence of configurations: $\hat{\mathbf{c}}_{i_{1}}, \hat{\mathbf{c}}_{i_{1}+1}, \ldots, \hat{\mathbf{c}}_{j_{1}}$, where $\hat{\mathbf{c}}_{p}$ is a restriction of $\mathbf{c}_{p}$ on the vertices of $P_{n-1}^{k}$ for $p \in\left[i_{1}, j_{1}\right]$. By the firing rule, as no vertices are fired on $\varepsilon_{1}$, for $p \in\left[i_{1}, j_{1}\right], \omega\left(\mathbf{c}_{p}\right)$ 's are constant, and $\mathbf{c}_{p}(v)$ 's are constant for each $v \in[k-2]$. Let $\hat{\omega}\left(\hat{\mathbf{c}}_{p}\right)=\sum_{v=k}^{n(k-1)} \mathbf{c}_{p}(v)$. As $\mathbf{c}_{i_{1}}(k-1)=\mathbf{c}_{j_{1}}(k-1)=k-1$, we have $\hat{\omega}\left(\hat{\mathbf{c}}_{i_{1}}\right)=\hat{\omega}\left(\hat{\mathbf{c}}_{j_{1}}\right)$, which implies that each vertex on $\varepsilon_{2}$ rather than $k-1$ will be fired exactly once by Lemma 4.2 (iii). So

$$
\mathbf{c}^{\prime}(k-1)=\mathbf{c}_{0}(k-1)
$$

By induction, we have $\hat{\mathbf{c}}_{i_{1}} \prec \hat{\mathbf{c}}_{j_{1}}$, and there exists a vertex $u>k-1$ such that $\hat{\mathbf{c}}_{i_{1}}(v)=\hat{\mathbf{c}}_{j_{1}}(v)$ for $k-1 \leq v \leq u$ and $\hat{\mathbf{c}}_{i_{1}}(u)>\hat{\mathbf{c}}_{j_{1}}(u)$. So, combining Eqs. (4.1), (4.3) and (4.2), we have $\mathbf{c}_{0}(v)=\mathbf{c}^{\prime}(v)$ for $1 \leq v \leq u$ and $\mathbf{c}_{0}(u)>\mathbf{c}^{\prime}(u)$, implying $\mathbf{c}_{0} \prec \mathbf{c}^{\prime}$.

\subsection{Formulas from firing graph}

Our goal is to compute the determinant of the multiplication map by $f_{w}$ of $A=$ $\frac{\mathbb{C}\left[x_{v}: v \in \widehat{V}\right]}{\left\langle f_{v}: v \in \widehat{V}\right\rangle}$ associated with the hyperpath $P_{n}^{k}$ as in Fig. 3, where $w$ is taken to be the vertex 0 . We focus on the firing graph $\mathcal{G}\left(\mathbf{c}_{0}\right)$ of the structure shown in Fig. 5. If a configuration $\mathbf{c}$ in $\mathcal{G}\left(\mathbf{c}_{0}\right)$ refers to a homogeneous polynomial $\mathbf{x}^{\mathbf{c}}=\prod_{\mathbf{c} \in \widehat{V}} x_{v}^{\mathbf{c}(v)}$ by ignoring the bank vertex, then we have

$$
m_{f_{0}}\left(\mathbf{x}^{\mathbf{c}_{0}}\right)=\lambda \mathbf{x}^{\mathbf{c}_{0}}-\mathbf{x}^{\widehat{\varepsilon}} \mathbf{x}^{\mathbf{c}_{0}}=\lambda \mathbf{x}^{\mathbf{c}_{0}}-\mathbf{x}^{\overline{\mathbf{c}}_{0}} .
$$


If there is a non-stable vertex $v$ in a configuration $\mathbf{c}$ with maximal order, then $v$ will be fired on each edge containing $v$, which yields configurations $\mathbf{c}^{\prime}$ and $\operatorname{arcs}\left(\mathbf{c}, \mathbf{c}^{\prime}\right)$. As $f_{v}=0$ inside $A, x_{v}^{k-1}=\frac{1}{\lambda}\left(\sum_{v \in \varepsilon \in E \backslash E_{w}} \mathbf{x}_{\varepsilon \backslash\{v\}}+\sum_{v \in \varepsilon \in E_{w}} \mathbf{x}_{e_{\hat{w}} \backslash\{v\}}\right)$. So we have

$$
\mathbf{x}^{\mathbf{c}}=\sum_{\left(\mathbf{c}, \mathbf{c}^{\prime}\right) \in \mathcal{E}\left(\mathcal{G}\left(\mathbf{c}_{0}\right)\right)} \frac{1}{\lambda} \mathbf{x}^{\mathbf{c}^{\prime}}
$$

for any $\mathbf{c} \neq \mathbf{c}_{0}$. Intuitively, a firing of the bank vertex means a multiplication of $f_{0}$, and a firing of other vertex $v$ means a substitution of $x_{v}^{k-1}$ by using $f_{v}=0$.

In view of this, we consider the weighted directed graph, still denoted by $\mathcal{G}\left(\mathbf{c}_{0}\right)$, by assigning the weight 1 on the arrow $\alpha_{0, \varepsilon_{1}}$ and the weight $\frac{1}{\lambda}$ on the others. In order to obtain more explicit formulas, we need simplify the weighted firing graph $\mathcal{G}\left(\mathbf{c}_{0}\right)$. Observe that the directed graph $\mathcal{G}\left(\mathbf{c}_{0}\right)$ may contain non-stable configurations. Next, we will erase all non-stable configurations by modifying the weight until all of vertices are stable configurations.

Define a function $g^{i}(x)$ in indeterminant $\lambda$ recursively:

$$
g^{-1}(x)=0, g^{0}(x)=1, g^{1}(x)=g(x)=\frac{1}{1-\frac{x}{\lambda^{k}}}, g^{i}(x)=g^{i-1}(g(x)) \text { for } i \geq 2 \text {. }
$$

Lemma 4.6 Let $\mathbf{c}_{0}$ be a configuration in $\mathcal{B}_{s}, 0 \leq s \leq n$. Then, we have

$$
\mathbf{x}^{\overline{\mathbf{c}}_{0}}=\frac{g^{S-1}(1)}{\lambda^{k-1}} \mathbf{x}^{\mathbf{c}_{0}}+\sum_{\mathbf{c}^{\prime} \in \mathcal{S}\left(\mathcal{G}^{\prime}\right)} h_{\mathbf{c}^{\prime}}(\lambda) \mathbf{x}^{\mathbf{c}^{\prime}}
$$

where $\overline{\mathbf{c}}_{0}(v)=\left\{\begin{array}{ll}\mathbf{c}_{0}(v)+1, & 1 \leq v \leq k-1, \\ \mathbf{c}_{0}(v), & k \leq v \leq n(k-1)\end{array}, g^{i}(x)\right.$ is defined as in $(4.4), h_{\mathbf{c}^{\prime}}(\lambda)$ is a function in $\lambda$ for each $\mathbf{c}^{\prime}$, and $\mathcal{S}\left(\mathcal{G}^{\prime}\right)$ is the set of all stable configurations in $\mathcal{G}^{\prime}$ defined in Proposition 4.5.

Proof Clearly, for $s=0$,

$$
\mathbf{x}^{\overline{\mathbf{c}}_{0}}=\sum_{\mathbf{c}^{\prime} \in \mathcal{S}\left(\mathcal{G}^{\prime}\right)} h_{\mathbf{c}^{\prime}}(\lambda) \mathbf{x}^{\mathbf{c}^{\prime}}=\frac{g^{s-1}(1)}{\lambda^{k-1}} \mathbf{x}^{\mathbf{c}_{0}}+\sum_{\mathbf{c}^{\prime} \in \mathcal{S}\left(\mathcal{G}^{\prime}\right)} h_{\mathbf{c}^{\prime}}(\lambda) \mathbf{x}^{\mathbf{c}^{\prime}}
$$

and for $s=1$,

$$
\mathbf{x}^{\overline{\mathbf{c}}_{0}}=\frac{1}{\lambda^{k-1}} \mathbf{x}^{\mathbf{c}_{0}}+\sum_{\mathbf{c}^{\prime} \in \mathcal{S}\left(\mathcal{G}^{\prime}\right)} h_{\mathbf{c}^{\prime}}(\lambda) \mathbf{x}^{\mathbf{c}^{\prime}}
$$

For $s \geq 2$, from the subgraph of $\mathcal{G}\left(\mathbf{c}_{0}\right)$ in Fig. 7, we get

$$
\mathbf{x}^{\mathbf{c}_{s-1}}=\frac{1}{\lambda} \mathbf{x}^{\overline{\mathbf{c}}_{s-1}}+\frac{1}{\lambda^{k}} \mathbf{x}^{\mathbf{c}_{s-1}}+\frac{1}{\lambda^{l_{s}}} \mathbf{x}^{\mathbf{c}_{1}^{\prime}},
$$


Fig. 7 Weighted $s$ th direct cycle by firing the vertices in $\varepsilon_{S}$

Fig. 8 Modified weighted $(s-1)$ th direct cycle by erasing the $s$ th directed cycle
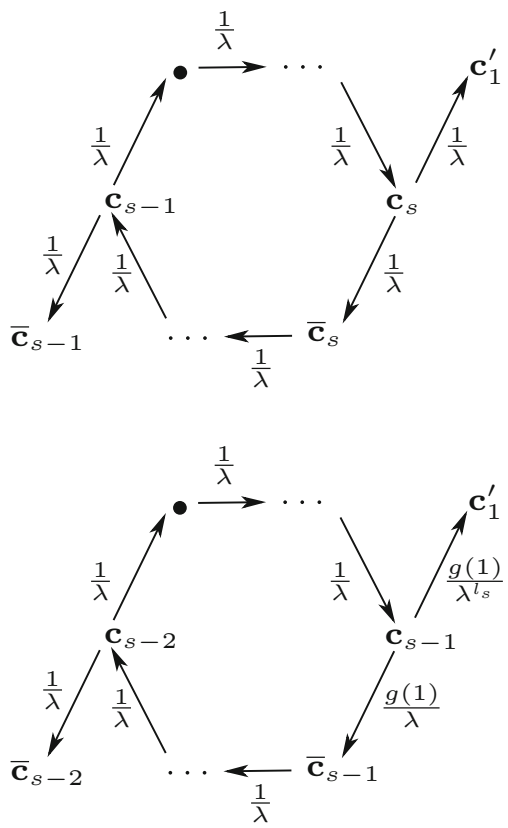

where $l_{s}$ is the length of the directed path from $\mathbf{c}_{s-1}$ to $\mathbf{c}_{1}^{\prime}$. It follows that

$$
\mathbf{x}^{\mathbf{c}_{s-1}}=g(1) \frac{1}{\lambda} \mathbf{x}^{\overline{\mathbf{c}}_{s-1}}+g(1) \frac{1}{\lambda^{l_{s}}} \mathbf{x}^{\mathbf{c}_{1}^{\prime}}
$$

Therefore, we can erase the $s$ th directed cycle by adding an arrow $\left(\mathbf{c}_{s-1}, \mathbf{c}_{1}^{\prime}\right)$ with weight $\frac{g(1)}{\lambda^{l_{s}}}$ and replacing the weight of $\left(\mathbf{c}_{s-1}, \overline{\mathbf{c}}_{s-1}\right)$ by $\frac{g(1)}{\lambda}$; see Fig. 8 .

Let $l_{j}$ be the length of the directed path from $\mathbf{c}_{j-1}$ to $\mathbf{c}_{j}$ for $2 \leq j \leq s-1$. From this modified weighted subgraph, we immediately get

$$
\mathbf{x}^{\mathbf{c}_{s-2}}=\frac{1}{\lambda} \mathbf{x}^{\overline{\mathbf{c}}_{s-2}}+g(1) \frac{1}{\lambda^{k}} \mathbf{x}^{\mathbf{c}_{s-2}}+g(1) \frac{1}{\lambda^{l_{s-1}+l_{s}}} \mathbf{x}^{\mathbf{c}_{1}^{\prime}},
$$

and

$$
\mathbf{x}^{\mathbf{c}_{s-2}}=g^{2}(1) \frac{1}{\lambda} \mathbf{x}^{\overline{\mathbf{c}}_{s-2}}+g^{2}(1) g(1) \frac{1}{\lambda^{l_{s-1}+l_{s}}} \mathbf{x}^{\mathbf{c}_{1}^{\prime}} .
$$

Assume that $\mathbf{x}^{\mathbf{c}_{s-i}}=g^{i}(1) \frac{1}{\lambda} \mathbf{x}^{\overline{\mathbf{c}}_{s-i}}+\frac{g^{i}(1) \cdots g^{2}(1) g(1)}{\lambda^{l_{s-i+1}+l_{s-i+2} \cdots+l_{s}}} \mathbf{x}^{\mathbf{c}_{1}^{\prime}}$ for $i \geq 1$. Then, from the modified weighted subgraph (Fig. 9), we have

$$
\mathbf{x}^{\mathbf{c}_{s-(i+1)}}=\frac{1}{\lambda} \mathbf{x}^{\overline{\mathbf{c}}_{s-(i+1)}}+g^{i}(1) \frac{1}{\lambda^{k}} \mathbf{x}^{\mathbf{c}_{s-(i+1)}}+\frac{g^{i}(1) \cdots g^{2}(1) g(1)}{\lambda^{l_{s-i}+l_{s-i+1}+\cdots+l_{s}}} \mathbf{x}^{\mathbf{c}_{1}^{\prime}},
$$


Fig. 9 Modified weighted $(s-i)$ th direct cycle by erasing the $(s-i+1)$ th, $\ldots, s$ th directed cycles

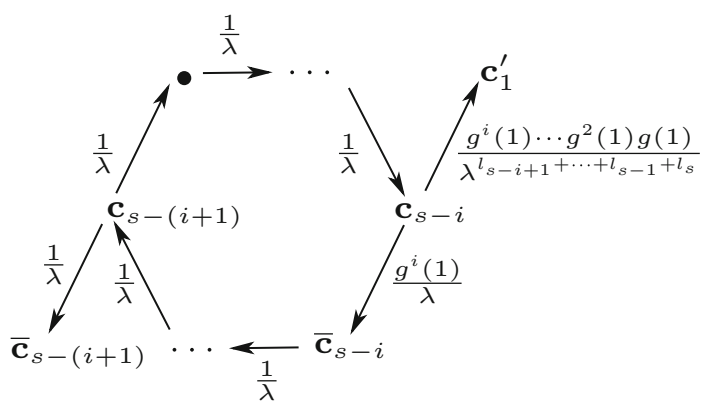

and hence

$$
\mathbf{x}^{\mathbf{c}_{s-(i+1)}}=g^{i+1}(1) \frac{1}{\lambda} \mathbf{x}^{\overline{\mathbf{c}}_{s-(i+1)}}+\frac{g^{i+1}(1) \cdots g^{2}(1) g(1)}{\lambda^{l_{s-i}+l_{s-i+1}+\cdots+l_{s}}} \mathbf{x}^{\mathbf{c}_{1}^{\prime}} .
$$

Taking $i=s-2$, we get

$$
\mathbf{x}^{\mathbf{c}_{1}}=g^{s-1}(1) \frac{1}{\lambda} \mathbf{x}^{\overline{\mathbf{c}}_{1}}+\frac{g^{s-1}(1) \cdots g^{2}(1) g(1)}{\lambda^{l_{2}+l_{3}+\cdots+l_{s}}} \mathbf{x}^{\mathbf{c}_{1}^{\prime}} .
$$

It follows that

$$
\mathbf{x}^{\overline{\mathbf{c}}_{0}}=\frac{g^{s-1}(1)}{\lambda^{k-1}} \mathbf{x}^{\mathbf{c}_{0}}+\prod_{i=1}^{s-1} g^{i}(1) \frac{1}{\lambda^{\sum_{j=1}^{s} l_{j}}} \mathbf{x}^{\mathbf{c}_{1}^{\prime}},
$$

where $l_{1}$ is the length of the directed path from $\overline{\mathbf{c}}_{0}$ to $\mathbf{c}_{1}$. By Proposition 4.5 (i), we know that there is not an arrow from $\mathcal{G}^{\prime}$ to $\mathcal{G}$ and therefore $\mathbf{x}^{\mathbf{c}_{1}^{\prime}}=\sum_{\mathbf{c}^{\prime} \in \mathcal{S}\left(\mathcal{G}^{\prime}\right)} \bar{h}_{\mathbf{c}^{\prime}}(\lambda) \mathbf{x}^{\mathbf{c}^{\prime}}$ for some function $\bar{h}_{\mathbf{c}^{\prime}}(\lambda)$ in $\lambda$. It follows that

$$
\mathbf{x}^{\overline{\mathbf{c}}_{0}}=\frac{g^{s-1}(1)}{\lambda^{k-1}} \mathbf{x}^{\mathbf{c}_{0}}+\sum_{\mathbf{c}^{\prime} \in \mathcal{S}\left(\mathcal{G}^{\prime}\right)} h_{\mathbf{c}^{\prime}}(\lambda) \mathbf{x}^{\mathbf{c}^{\prime}}
$$

where $h_{\mathbf{c}^{\prime}}(\lambda)=\prod_{i=1}^{s-1} g^{i}(1) \frac{1}{\lambda^{\sum_{j=1}^{s} l_{j}}} \bar{h}_{\mathbf{c}^{\prime}}(\lambda)$.

\subsection{The characteristic polynomial of a hyperpath}

We will give a recursive formula of the characteristic polynomial of hyperpaths. Observe the number of stable configurations on $K_{k}$ is $(k-1)^{k-1}$, and the number of critical configurations on $K_{k}$ is $k^{k-2}$ by Example 2.8. So $\mathcal{B}_{s}$ contains exactly $\mu_{n, k}(s)$ elements, which is defined as follows:

$$
\mu_{n, k}(s)= \begin{cases}k^{s(k-2)}\left((k-1)^{k-1}-k^{k-2}\right)(k-1)^{(n-s-1)(k-1)}, & s \in[0, n-1] \\ k^{n(k-2)}, & s=n\end{cases}
$$


Theorem 4.7 Let $\phi_{n, k}^{P}(\lambda)$ be the characteristic polynomial of the hyperpath $P_{n}^{k}$, where $n \geq 2$. Then,

$$
\phi_{n, k}^{P}(\lambda)=\lambda^{(k-2)(k-1)^{n(k-1)}} \prod_{s=0}^{n}\left(\lambda-\frac{g^{s-1}(1)}{\lambda^{k-1}}\right)^{\mu_{n, k}(s)} \phi_{n-1, k}^{P}(\lambda)^{(k-1)^{k-1}}
$$

where $g^{i}(x)$ is defined in (4.4) and $\mu_{n, k}(s)$ is defined in (4.5).

Proof By definition, the characteristic polynomial of $P_{n}^{k}$ is

$$
\phi_{n, k}^{P}(\lambda)=\operatorname{Res}\left(F_{0}, F_{1}, \ldots, F_{r}\right)
$$

where

$$
F_{i}\left(x_{0}, x_{1}, \ldots, x_{r}\right)=\lambda x_{i}^{k-1}-\sum_{i \in \varepsilon \in E} \mathbf{x}^{\varepsilon \backslash\{i\}}
$$

is a homogeneous polynomial in variables $x_{0}, x_{1}, \ldots, x_{r}$ of degree $k-1$ for $i=$ $0,1, \ldots, r=n(k-1)$. Clearly,

$$
f_{i}\left(x_{1}, \ldots, x_{r}\right)= \begin{cases}\lambda-x_{1} \cdots x_{k-1}, & \text { if } i=0, \\ \lambda x_{i}^{k-1}-x_{1} \cdots x_{i-1} x_{i+1} \cdots x_{k-1}, & \text { if } i \in[k-2], \\ \lambda x_{k-1}^{k-1}-x_{1} \cdots x_{k-2}-x_{k} \cdots x_{2(k-1)}, & \text { if } i=k-1, \\ F_{i}\left(x_{0}, x_{1}, \ldots, x_{r}\right), & \text { otherwise }\end{cases}
$$

and

$$
\bar{F}_{i}\left(x_{1}, \ldots, x_{r}\right)= \begin{cases}\lambda x_{i}^{k-1}, & \text { if } i \in[k-2] \\ \lambda x_{k-1}^{k-1}-x_{k} \cdots x_{2(k-1)}, & \text { if } i=k-1 \\ F_{i}\left(x_{0}, x_{1}, \ldots, x_{r}\right), & \text { otherwise }\end{cases}
$$

By Lemmas 2.2 and 2.3, we have

$$
\begin{aligned}
\phi_{n, k}^{P}(\lambda)= & \operatorname{Res}\left(\bar{F}_{1}, \ldots, \bar{F}_{r}\right)^{k-1} \operatorname{det}\left(m_{f_{0}}\right) \\
= & \operatorname{Res}\left(\lambda x_{1}^{k-1}, \ldots, \lambda x_{k-2}^{k-1}, \bar{F}_{k-1}, \ldots, \bar{F}_{r}\right)^{k-1} \operatorname{det}\left(m_{f_{0}}\right) \\
= & \left(\operatorname{Res}\left(\lambda x_{1}^{k-1}, \ldots, \lambda x_{k-2}^{k-1}\right)^{(k-1)^{(n-1)(k-1)+1}} \operatorname{Res}\left(\bar{F}_{k-1}, \ldots, \bar{F}_{r}\right)^{(k-1)^{k-2}}\right)^{k-1} \\
& \times \operatorname{det}\left(m_{f_{0}}\right) \\
= & \lambda^{(k-2)(k-1)^{n(k-1)}} \phi_{n-1, k}^{P}(\lambda)^{(k-1)^{k-1}} \operatorname{det}\left(m_{f_{0}}\right),
\end{aligned}
$$

where $m_{f_{0}}$ is the multiplication map of the quotient algebra

$$
A=\mathbb{C}\left[x_{1}, \ldots, x_{r}\right] /\left\langle f_{1}, \ldots, f_{r}\right\rangle
$$

given by $m_{f_{0}}\left(x_{1}^{i_{1}} \cdots x_{r}^{i_{r}}\right)=\lambda x_{1}^{i_{1}} \cdots x_{r}^{i_{r}}-x_{1}^{i_{1}+1} \cdots x_{k-1}^{i_{k-1}+1} x_{k}^{i_{k}} \cdots x_{r}^{i_{r}}$. 
We choose a $\mathbb{C}$-basis $\mathbf{B}=\left\{\mathbf{x}^{\mathbf{c}} \mid \mathbf{c}:[r] \rightarrow[k-2]\right\}$ for $A$, where $\mathbf{x}^{\mathbf{c}}=x_{1}^{\mathbf{c}(1)} \cdots x_{r}^{\mathbf{c}(r)}$. In fact, for any $\mathbf{x}^{\mathbf{c}} \in \mathbf{B}, \tilde{\mathbf{c}}=(\tilde{\mathbf{c}}(0), \mathbf{c}(1), \ldots, \mathbf{c}(r))$ can be viewed as a stable configuration on $P_{n}^{k}$, where 0 is the bank vertex whose value can be omitted. We denote by $\mathcal{B}$ the set of all stable configurations on $P_{n}^{k}$. Clearly, there is a one-to-one correspondence between $\mathbf{B}$ and $\mathcal{B}$ by ignoring the bank vertex, and the left anti-lexicographical ordering on $\mathcal{B}$ gives a total ordering on $\mathbf{B}$. To be precise, $x_{1}^{i_{1}} \cdots x_{r}^{i_{r}} \prec x_{1}^{j_{1}} \cdots x_{r}^{j_{r}}$ if and only if $\sum_{t=1}^{r} i_{t}<\sum_{t=1}^{r} j_{t}$, or $\sum_{t=1}^{r} i_{t}=\sum_{t=1}^{r} j_{t}, i_{s}=j_{s}$ for $1 \leq s \leq t-1$ and $i_{t}>j_{t}$ for some $t \in[r]$.

Retaining the notation in Sect. 4.2, we observe that $\mathcal{B}$ is exactly the disjoint union of $\mathcal{B}_{0}, \mathcal{B}_{1}, \ldots, \mathcal{B}_{n}$. For each $s \in[n]$, the number of configurations in $\mathcal{B}_{s}$ is exactly $\mu_{n, k}(s)$. Let $\mathbf{c}_{0}$ be a configuration in $\mathcal{B}_{s}$. By Lemma 4.6, we know

$$
m_{f_{0}}\left(\mathbf{x}^{\mathbf{c}_{0}}\right)=\mathbf{x}^{\overline{\mathbf{c}}_{0}}=\left(\lambda-\frac{g^{s-1}(1)}{\lambda^{k-1}}\right) \mathbf{x}^{\mathbf{c}_{0}}-\sum_{\mathbf{c}^{\prime} \in \mathcal{S}\left(\mathcal{G}^{\prime}\right)} h_{\mathbf{c}^{\prime}}(\lambda) \mathbf{x}^{\mathbf{c}^{\prime}}
$$

where $\mathcal{G}^{\prime}, \mathcal{S}\left(\mathcal{G}^{\prime}\right)$ and $h_{\mathbf{c}^{\prime}}(\lambda)$ are defined in Proposition 4.5 or Lemma 4.6. On the other hand, by Proposition 4.5 (ii), we have $\mathbf{c}_{0} \prec \mathbf{c}^{\prime}$ for any $\mathbf{c}^{\prime}$ in $\mathcal{G}^{\prime}$. It follows that the matrix of $m_{f_{0}}$ associated with the ordered basis $\mathbf{B}$ is a lower triangle matrix with $\frac{g^{s-1}(1)}{\lambda^{k-1}}$ appearing on the diagonal exactly $\mu_{n, k}(s)$ times for $s \in[0, n]$. So

$$
\operatorname{det}\left(m_{f_{0}}\right)=\prod_{s=0}^{n}\left(\lambda-\frac{g^{s-1}(1)}{\lambda^{k-1}}\right)^{\mu_{n, k}(s)}
$$

and

$$
\phi_{n, k}^{P}(\lambda)=\lambda^{(k-2)(k-1)^{n(k-1)}} \prod_{s=0}^{n}\left(\lambda-\frac{g^{s-1}(1)}{\lambda^{k-1}}\right)^{\mu_{n, k}(s)} \phi_{n-1, k}^{P}(\lambda)^{(k-1)^{k-1}} .
$$

Example 4.8 By Theorem 4.7, we can deduce the characteristic polynomial of $P_{n}^{k}$ inductively from an edge. The following are the characteristic polynomials $\phi_{n, k}^{P}(\lambda)$ of $P_{n}^{k}$ for some specified $n$ and $k$.

$$
\begin{aligned}
& \phi_{1,3}^{P}(\lambda)=\lambda^{3}\left(\lambda^{3}-1\right)^{3} \text { of degree } 12 \\
& \phi_{2,3}^{P}(\lambda)=\lambda^{35}\left(\lambda^{3}-1\right)^{6}\left(\lambda^{3}-2\right)^{9} \text { of degree } 80 \\
& \phi_{3,3}^{P}(\lambda)=\lambda^{151}\left(\lambda^{3}-1\right)^{27}\left(\lambda^{3}-2\right)^{18}\left(\lambda^{6}-3 \lambda^{3}+1\right)^{27} \text { of degree } 448 \\
& \phi_{4,3}^{P}(\lambda)=\lambda^{891}\left(\lambda^{3}-1\right)^{201}\left(\lambda^{3}-2\right)^{81}\left(\lambda^{3}-3\right)^{81}\left(\lambda^{6}-3 \lambda^{3}+1\right)^{54} \text { of degree } 2304 \\
& \phi_{1,4}^{P}(\lambda)=\lambda^{44}\left(t^{4}-1\right)^{16} \text { of degree } 108 \\
& \phi_{2,4}^{P}(\lambda)=\lambda^{2671}\left(\lambda^{4}-1\right)^{352}\left(\lambda^{4}-2\right)^{256} \text { of degree } 5103 ; \\
& \phi_{3,4}^{P}(\lambda)=\lambda^{95774}\left(\lambda^{4}-1\right)^{11440}\left(\lambda^{4}-2\right)^{5632}\left(\lambda^{8}-3 \lambda^{4}+1\right)^{4096} \text { of degree } 196830
\end{aligned}
$$


Fig. 10 Starlike hypergraph $S_{n_{1}, n_{2}, \ldots, n_{m}}^{k}$

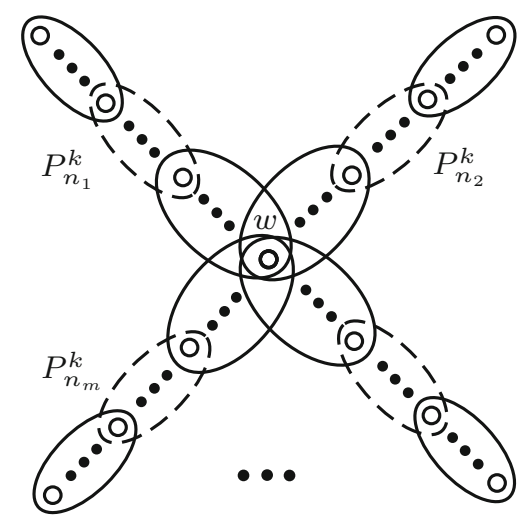

\section{Starlike hypergraphs}

In this section, we will deal with a class of $k$-uniform hypergraphs, called starlike hypergraph and denoted by $S_{n_{1}, \ldots, n_{m}}^{k}$, which is obtained from $m$ hyperpaths of length $n_{1}, \ldots, n_{m}$ by sharing a common vertex $w$, where $m \geq 1$ and $n_{i} \geq 1$ for $i \in[m]$; see Fig. 10. When $m=1$, it is a hyperpath $P_{n_{1}}^{k}$. When $n_{i}=1$ for each $i \in[m]$, it is called a hyperstar with $m$ edges and denoted by $S_{m}^{k}$.

Theorem 5.1 Let $S_{n_{1}, \ldots, n_{m}}^{k}$ be the k-uniform starlike hypergraph with exactly thyperpaths having length 1 , where $0 \leq t \leq m$. Then, the characteristic polynomial $\phi_{n_{1}, \ldots, n_{m} ; k}^{S H}(\lambda)$ of $S_{n_{1}, \ldots, n_{m}}^{k}$ is

$$
\begin{gathered}
\lambda^{(m(k-2)+t)(k-1)^{\sum_{j=1}^{m} r_{j}}} \prod_{\substack{i \in[m] \\
n_{i}>1}} \phi_{n_{i}-1, k}^{P}(\lambda)^{(k-1)^{k-1+\sum_{j \neq i} r_{j}}} \\
\times \prod_{\substack{s_{i} \in\left[0, n_{i}\right] \\
1 \leq i \leq m}}\left(\lambda-\sum_{i=1}^{m} \frac{g^{s_{i}-1}(1)}{\lambda^{k-1}}\right)^{\prod_{i=1}^{m} \mu_{n_{i}, k}\left(s_{i}\right)}
\end{gathered}
$$

where $r_{i}=n_{i}(k-1)$ for $i \in[m], \phi_{n, k}^{P}(\lambda)$ denotes the characteristic polynomial of $P_{n}^{k}, g^{s}(x)$ and $\mu_{n, k}(s)$ are defined in (4.4) and (4.5), respectively.

Proof For each $i \in[m]$, let $\varepsilon_{i}$ be the edge of $P_{n_{i}}^{k}$ containing $w$, and $\widehat{\varepsilon}_{i}=\varepsilon_{i} \backslash\{w\}$. Let $H_{i}$ be sub-hyperpath of $P_{n_{i}}^{k}$ by deleting the vertex $w$ and the edge $\varepsilon_{i}$ for $i \in[m]$, which is a hyperpath $P_{n_{i}-1}^{k}$ together with $k-2$ isolated vertices if $n_{i}>1$ or $r_{i}(=k-1)$ isolated vertices otherwise. If $n_{i}>1$, by Lemmas 2.2 and 2.3, we get

$$
\phi_{H_{i}}(\lambda)=\lambda^{(k-2)(k-1)^{r_{i}-1}} \phi_{n_{i}-1, k}^{P}(\lambda)^{(k-1)^{k-2}}
$$


otherwise,

$$
\phi_{H_{i}}(\lambda)=\lambda^{(k-1)(k-1)^{r_{i}-1}} .
$$

So, combining (5.1) and (5.2), we have

$$
\prod_{i=1}^{m} \phi_{H_{i}}^{(k-1)^{1+\sum_{j \neq i} r_{j}}}=\lambda^{(m(k-2)+t)(k-1)^{\sum_{j=1}^{m} r_{j}}} \prod_{\substack{i \in[m] \\ n_{i}>1}} \phi_{n_{i}-1, k}^{P}(\lambda)^{(k-1)^{k-1+\sum_{j \neq i} r_{j}}} .
$$

Let $V$ be the vertex set of $S_{n_{1}, \ldots, n_{m}}^{k}$, and let $V_{i}$ be the vertex set of $P_{n_{i}}^{k}$ for $i \in[m]$. Note that $w$ is a cut vertex of $S_{n_{1}, \ldots, n_{m}}^{k}$ and recall the discussion in Sect. 3.2. The quotient algebra $A=\frac{\mathbb{C}\left[x_{v}: v \in V \backslash\{w\}\right]}{\left\langle f_{v}: v \in V \backslash\{w\}\right\rangle}$ holds

$$
A=A_{1} \otimes \cdots \otimes A_{m}
$$

where $A_{i}=\frac{\mathbb{C}\left[x_{v}: v \in V_{i} \backslash\{w\}\right]}{\left\langle f_{v}: v \in V_{i} \backslash\{w\}\right\rangle}$ for $i \in[m]$, and

$$
f_{v}=\lambda x_{v}^{k-1}-\sum_{v \in \varepsilon \in E\left(P_{n_{i}}^{k}\right)} x_{\varepsilon \backslash\{v\}}-\sum_{v \in \widehat{\varepsilon}_{i}} x_{\widehat{\varepsilon}_{i} \backslash\{v\}}, v \in V_{i} \backslash\{w\}, i \in[m] .
$$

Let

$$
f_{w}=\lambda-\sum_{i=1}^{m} x_{\widehat{\varepsilon}_{i}}
$$

and $m_{f_{w}}: A \rightarrow A$ be the linear map given by multiplication by $f_{w}$. Then,

$$
m_{f_{w}}=\lambda \operatorname{Id}_{A}-\sum_{i=1}^{m} \operatorname{Id}_{A_{1}} \otimes \cdots \otimes \operatorname{Id}_{A_{i-1}} \otimes m_{i, w} \otimes \operatorname{Id}_{A_{i+1}} \otimes \cdots \otimes \operatorname{Id}_{A_{m}},
$$

where $m_{i, w}: A_{i} \rightarrow A_{i}$ is the linear map given by multiplication by $\mathbf{x}_{\widehat{\varepsilon}_{i}}$ for $i \in[m]$.

Fix an $i \in[m]$. Let $\mathbf{B}=\left\{\mathbf{x}^{\mathbf{c}} \mid \mathbf{c}: V_{i} \backslash\{w\} \rightarrow[k-2]\right\}$ be a basis of $A_{i}$, where $\mathbf{x}^{\mathbf{c}}=\prod_{v \in V_{i} \backslash\{w\}} x_{v}^{\mathbf{c}(v)}$, and let $\mathcal{B}$ be the set of stable configuration of $P_{n_{i}}^{k}$ with $w$ as the bank vertex. As discussed in Theorem 4.7, there is a one-to-one correspondence between $\mathbf{B}$ and $\mathcal{B}$ by ignoring the bank vertex. We also have a left anti-lexicographical ordering $\prec$ on $\mathbf{B}$ arising from the order of $\mathcal{B}$. Retaining the notation in Sect. 4.2, $\mathcal{B}$ is the disjoint union of $\mathcal{B}_{0}, \mathcal{B}_{1}, \ldots, \mathcal{B}_{n_{i}}$, and the number of configurations in $\mathcal{B}_{S}$ is $\mu_{n_{i}, k}(s)$ for each $s \in\left[0, n_{i}\right]$. 
For each $\mathbf{c}_{0} \in \mathcal{B}_{s}$, by Lemma 4.6,

$$
m_{i, w}\left(\mathbf{x}^{\mathbf{c}_{0}}\right)=\mathbf{x}^{\overline{\mathbf{c}}_{0}}=\frac{g^{s-1}(1)}{\lambda^{k-1}} \mathbf{x}^{\mathbf{c}_{0}}+\sum_{\mathbf{c}^{\prime} \in \mathcal{S}\left(\mathcal{G}^{\prime}\right)} h_{\mathbf{c}^{\prime}}(\lambda) \mathbf{x}^{\mathbf{c}^{\prime}},
$$

where $\mathcal{G}^{\prime}, \mathcal{S}\left(\mathcal{G}^{\prime}\right)$ and $h_{\mathbf{c}^{\prime}}(\lambda)$ are defined in Proposition 4.5 or Lemma 4.6. Note that $\mathbf{c}_{0} \prec \mathbf{c}^{\prime}$ for any $c^{\prime} \in \mathcal{G}^{\prime}$. So the matrix of $m_{i, w}$ associated with the basis $\mathbf{B}$ under the above order is a lower triangle matrix with $\frac{g^{s-1}(1)}{\lambda^{k-1}}$ appearing on the diagonal exactly $\mu_{n_{i}, k}(s)$ times for $s \in\left[0, n_{i}\right]$.

By the above discussion,

$$
\operatorname{det}\left(m_{f_{w}}\right)=\prod_{\substack{s_{i} \in\left[0, n_{i}\right] \\ 1 \leq i \leq m}}\left(\lambda-\sum_{i=1}^{m} \frac{g^{s_{i}-1}(1)}{\lambda^{k-1}}\right)^{\prod_{i=1}^{m} \mu_{n_{i}, k}\left(s_{i}\right)}
$$

The result follows by Corollary 3.2 and the equalities (5.3) and (5.4).

Taking $n_{i}=1$ for $i \in[m]$ in Theorem 5.1, we get the characteristic polynomial of hyperstar $S_{m}^{k}$.

Corollary 5.2 Let $\phi_{n, k}^{S}$ be the characteristic polynomial of the k-uniform hyperstar $S_{m}^{k}$ with $m$ edges. Then,

$$
\phi_{m, k}^{S}(\lambda)=\lambda^{r(k-1)^{r}} \prod_{p=0}^{m}\left(\lambda-\frac{p}{\lambda^{k-1}}\right)^{\left(\begin{array}{c}
m \\
p
\end{array}\right) k^{(k-2) p}\left((k-1)^{k-1}-k^{k-2}\right)^{n-p}}
$$

where $r=m(k-1)$.

Corollary 5.3 [5, Theorem 4.3] Let E be the k-uniform hypergraph with $k$ vertices and a single edge. Then,

$$
\phi_{E}(\lambda)=\lambda^{k(k-1)^{k-1}-k^{k-1}}\left(\lambda^{k}-1\right)^{k^{k-2}} .
$$

Corollary 5.4 Let $S_{2}^{k}$ be a k-uniform hyperstar with two edges or hyperpath with two edges. Then,

$$
\phi_{S_{2}^{k}}(\lambda)=\lambda^{\mu_{k}}\left(\lambda^{k}-1\right)^{2 k^{k-2}\left((k-1)^{k-1}-k^{k-2}\right)}\left(\lambda^{k}-2\right)^{k^{2(k-2)}},
$$

where $\mu_{k}=(2 k-1)(k-1)^{2(k-1)}-2 k^{k-1}(k-1)^{k-1}+k^{2 k-3}$. 
Corollary 5.5 The characteristic polynomial of the starlike hypergraph $S_{1,1,2}^{k}$ is

$$
\begin{aligned}
& \phi_{1,1,2 ; k}^{S H}(\lambda)=\lambda^{(4 k-3)(k-1)^{4(k-1)}-\left(k^{k-1}+3 k^{k-2}\right)(k-1)^{3(k-1)}+3 k^{2(k-2)}(k-1)^{2(k-1)}-k^{3(k-2)}(k-1)^{k-1}} \\
& \cdot\left(\lambda^{k}-1\right)^{k^{k-2}(k-1)^{3(k-1)}} \\
& \cdot\left(\lambda-\frac{1}{\lambda^{k-1}}\right)^{k^{k-2}\left(3(k-1)^{k-1}-k^{k-2}\right)\left((k-1)^{k-1}-k^{k-2}\right)^{2}} \\
& \cdot\left(\lambda-\frac{2}{\lambda^{k-1}}\right)^{k^{2(k-2)}\left(3(k-1)^{k-1}-2 k^{k-2}\right)\left((k-1)^{k-1}-k^{k-2}\right)} \\
& \cdot\left(\lambda-\frac{3}{\lambda^{k-1}}\right)^{k^{3(k-2)}\left((k-1)^{k-1}-k^{k-2}\right)} \\
& \cdot\left(\lambda-\frac{\lambda}{\lambda^{k}-1}\right)^{k^{2(k-2)}\left((k-1)^{k-1}-k^{k-2}\right)^{2}} \\
& \cdot\left(\lambda-\frac{1}{\lambda^{k-1}}-\frac{\lambda}{\lambda^{k}-1}\right)^{2 k^{3(k-2)}\left((k-1)^{k-1}-k^{k-2}\right)} \\
& \cdot\left(\lambda-\frac{2}{\lambda^{k-1}}-\frac{\lambda}{\lambda^{k}-1}\right)^{k^{4(k-2)}} \text {. }
\end{aligned}
$$

In particular, the characteristic polynomial $\phi_{1,1,2 ; 3}^{S H}(\lambda)$ of $S_{1,1,2}^{3}$ is

$$
\lambda^{980}\left(\lambda^{3}-1\right)^{75}\left(\lambda^{3}-2\right)^{54}\left(\lambda^{3}-3\right)^{27}\left(\lambda^{4}-2 \lambda\right)^{9}\left(\lambda^{6}-3 \lambda^{3}+1\right)^{54}\left(\lambda^{6}-4 \lambda^{3}+2\right)^{81}
$$

of degree 2294.

\section{Conclusion}

We give an explicit and recursive formula for the characteristic polynomial of the adjacency tensor of a starlike hypergraph, which is a resultant of a system of polynomials related to the structure of the hypergraph. Variants of the chip-firing game on simple graphs such as dollar game on simple graphs or hypergraphs are applied to analyze these resultants. As such, we provide a combinatorial method for computing resultants, which will have potential use for commutative algebra, algebraic geometry and physical fields.

We note that there are many numerical methods and algorithms for computing partial (real or extreme) eigenvalues of a general (symmetric) tensor; see e.g., Chang et al. [3], Chen et al. [4], Cui et al. [8] and the references therein. We also note the starlike hypergraph is a power hypergraph $G^{k}$, which is obtained from a starlike simple graph $G$ by adding $k-2$ vertices to each of its edges. Zhou et al. [21] proved that if $\lambda$ is a nonzero eigenvalue of $G$ or any subgraph of $G$, then $\lambda \frac{2}{k}$ is an eigenvalue of $G^{k}$. In fact, the nonzero eigenvalues of $G^{k}$ are exactly those eigenvalues arising from $G$ 
in the above way. Our method allows for calculating these same eigenvalues, with the added benefit of determining the algebraic multiplicity of each eigenvalue.

Acknowledgements The authors would like to thank the referees for patient reading of the paper and for giving valuable suggestions to improve the paper.

\section{References}

1. Anokhina, A.S., Morozov, A.Yu., Shakirov, Sh.R.: Resultant as the determinant of a Koszul complex. Theor. Math. Phys. 160(3), 1203-1228 (2009)

2. Biggs, N.L.: Chip-firing and the critical group of a graph. J. Algebraic Combin. 9(1), $25-45$ (1999)

3. Chang, J., Chen, Y., Qi, L.: Computing eigenvalues of large scale sparse tensors arising from a hypergraph. SIAM J. Sci. Comput. 38(6), A3618-A3643 (2016)

4. Chen, L., Han, L., Zhou, L.: Computing tensor eigenvalues via homotopy methods. SIAM J. Matrix Anal. Appl. 37(1), 290-319 (2016)

5. Cooper, J., Dutle, A.: Spectra of uniform hypergraphs. Linear Algebra Appl. 436(9), 3268-3292 (2012)

6. Cooper, J., Dutle, A.: Computing hypermatrix spectra with the Poisson product formula. Linear Multilinear Algebra 63(5), 956-970 (2015)

7. Cox, D.A., Little, J., O'Shea, D.: Using Algebraic Geometry, 2nd edn. Graduate Texts in Mathematics, vol 185. Springer, New York (2005)

8. Cui, C.-F., Dai, Y.-H., Nie, J.: All real eigenvalues of symmetric tensors. SIAM J. Matrix Anal. Appl. 35(4), 1582-1601 (2014)

9. Fan, Y.-Z., Huang, T., Bao, Y.-H., Zhuan-Sun, C.-L., Li, Y.-P.: The spectral symmetry of weakly irreducible nonnegative tensors and connected hypergraphs. Trans. Amer. Math. Soc. 372(3), 22132233 (2019)

10. Fan, Y.-Z., Bao, Y.-H., Huang, T.: Eigenvariety of nonnegative symmetric weakly irreducible tensors associated with spectral radius and its application to hypergraphs. Linear Algebra Appl. 564, 72-94 (2019)

11. Gelfand, I.M., Kapranov, M.M., Zelevinsky, A.V.: Discriminants, Resultants, and Multidimensional Determinants. Mathematics: Theory \& Applications, Mathematics. Birkhäuser, Boston (1994)

12. Hu, S., Huang, Z.-H., Ling, C., Qi, L.: On determinants and eigenvalue theory of tensors. J. Symbolic Comput. 50, 508-531 (2013)

13. Jouanolou, J.-P.: Le formalisme du résultant. Adv. Math. 90(2), 117-263 (1991)

14. Lim, L.-H.: Singular values and eigenvalues of tensors: a variational approach. In: Proceedings of the 1st IEEE International Workshop on Computational Advances in Multi-Sensor Adaptive Processing, vol. 1, pp. 129-132 (2005)

15. Morozov, A., Shakirov, Sh.: Analogue of the identity Log Det = Trace Log for resultants. J. Geom. Phys. 61(3), 708-726 (2011)

16. Morozov, A.Yu., Shakirov, Sh.R.: New and old results in resultant theory. Theor. Math. Phys. 163(2), 587-617 (2010)

17. Qi, L.: Eigenvalues of a real supersymmetric tensor. J. Symbolic Comput. 40(6), 1302-1324 (2005)

18. Qi, L.: Eigenvalues and invariants of tensors. J. Math. Anal. Appl. 325(2), 1363-1377 (2007)

19. Shao, J.-Y., Qi, L., Hu, S.: Some new trace formulas of tensors with applications in spectral hypergraph theory. Linear Multilinear Algebra 63(5), 971-992 (2015)

20. Shao, J.-Y., Shan, H.-Y., Wu, B.-F.: Some spectral properties and characterizations of connected oddbipartite uniform hypergraphs. Linear Multilinear Algebra 63(12), 2359-2372 (2015)

21. Zhou, J., Sun, L., Wang, W., Bu, C.: Some spectral properties of uniform hypergraphs. Electron. J. Combin. 21(4), Paper 4.24, 14 (2014)

Publisher's Note Springer Nature remains neutral with regard to jurisdictional claims in published maps and institutional affiliations. 